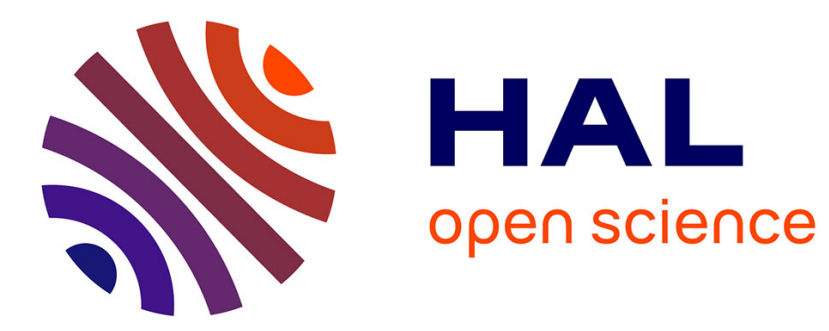

\title{
Magnetic Reconnection Inside a Flux Transfer Event-Like Structure in Magnetopause Kelvin-Helmholtz Waves
}

\author{
R. Kieokaew, B. Lavraud, C. Foullon, S. Toledo-redondo, N. Fargette, K.-j. \\ Hwang, K. Malakit, D. Ruffolo, M. Øieroset, T.-d. Phan, et al.
}

\section{To cite this version:}

R. Kieokaew, B. Lavraud, C. Foullon, S. Toledo-redondo, N. Fargette, et al.. Magnetic Reconnection Inside a Flux Transfer Event-Like Structure in Magnetopause Kelvin-Helmholtz Waves. Journal of Geophysical Research Space Physics, 2020, 125 (6), 10.1029/2019JA027527 . hal-02886338

\section{HAL Id: hal-02886338 https://hal.science/hal-02886338}

Submitted on 2 Jul 2020

HAL is a multi-disciplinary open access archive for the deposit and dissemination of scientific research documents, whether they are published or not. The documents may come from teaching and research institutions in France or abroad, or from public or private research centers.
L'archive ouverte pluridisciplinaire HAL, est destinée au dépôt et à la diffusion de documents scientifiques de niveau recherche, publiés ou non, émanant des établissements d'enseignement et de recherche français ou étrangers, des laboratoires publics ou privés. 


\section{Magnetic Reconnection inside a Flux Transfer Event-like structure in Magnetopause Kelvin-Helmholtz Waves}

R. Kieokaew ${ }^{1,2}$, B. Lavraud ${ }^{1}$, C. Foullon ${ }^{2}$, S. Toledo-Redondo ${ }^{1}$, N. Fargette ${ }^{1}$, K.-J. Hwang ${ }^{3}$, K. Malakit ${ }^{4}$, D. Ruffolo ${ }^{5}$, M. Øieroset ${ }^{6}$, T. D. Phan $^{6}$, H. Hasegawa $^{7}$, S. Fadanelli ${ }^{1,8}$, L. Avanov ${ }^{9,10}$, J. Burch ${ }^{3}$, D. J. Gershman ${ }^{9,10}$, B. Giles $^{9}$, J. Dorelli ${ }^{9}$, V. Génot ${ }^{1}$, C. Jacquey ${ }^{1}$, T. Moore ${ }^{9}$, W. Paterson ${ }^{9}$, C. Pollock $^{9}$, A. Rager ${ }^{9}$, Y. Saito ${ }^{7}$, J.-A. Sauvaud ${ }^{1}$, C. Schiff ${ }^{9}$, Y. Vernisse ${ }^{1}$, and E. Penou $^{1}$

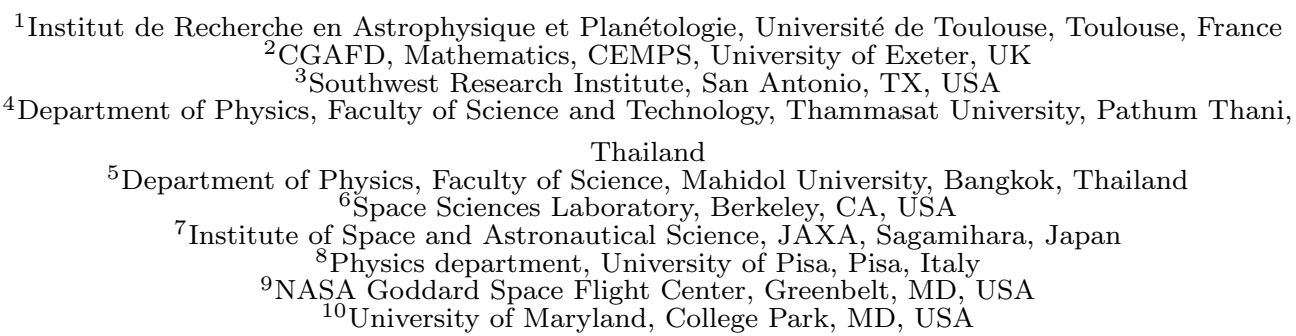

\section{Key Points:}

- MMS observation of a Flux Transfer Event type structure during a KelvinHelmholtz event on the dawn flank magnetopause beyond the terminator

- Its characteristics are consistent with a structure produced from multiple vortexinduced reconnection X-lines

- Reconnection signatures are found at the central current sheet while properties on either side indicate two flux tubes that interlace 


\begin{abstract}
Magnetopause Kelvin-Helmholtz (KH) waves are believed to mediate solar wind plasma transport via small-scale mechanisms. Vortex-induced reconnection (VIR) was predicted in simulations and recently observed using NASA's Magnetospheric Multiscale (MMS) mission data. Flux Transfer Events (FTEs) produced by VIR at multiple locations along the periphery of $\mathrm{KH}$ waves were also predicted in simulations but detailed observations were still lacking. Here we report MMS observations of an FTE-type structure in a KH wave trailing edge during KH activity on 5 May 2017 on the dawnside flank magnetopause. The structure is characterised by (1) bipolar magnetic $B_{Y}$ variation with enhanced core field $\left(B_{Z}\right)$ and $(2)$ enhanced total pressure with dominant magnetic pressure. The cross-section size of the FTE is found to be consistent with vortex-induced flux ropes predicted in the simulations. Unexpectedly, we observe an ion jet $\left(V_{Y}\right)$, electron parallel heating, ion and electron density enhancements, and other signatures that can be interpreted as a reconnection exhaust at the FTE central current sheet. Moreover, pitch angle distributions of suprathermal electrons on either side of the current sheet show different properties, indicating different magnetic connectivities. This FTE-type structure may thus alternatively be interpreted as two interlaced flux tubes with reconnection at the interface as reported by Kacem et al. (2018) and Øieroset et al. (2019). The structure may be the result of interaction between two flux tubes, likely produced by multiple VIR at the $\mathrm{KH}$ wave trailing edge, and constitutes a new class of phenomenon induced by KH waves.
\end{abstract}

\title{
1 Introduction
}

Kelvin-Helmholtz $(\mathrm{KH})$ waves are believed to facilitate solar wind plasma transport along the Earth's magnetopause and low-latitude boundary layer (LLBL) under northward interplanetary magnetic field (IMF) conditions. The waves are induced by magnetic KH instability due to a shear flow created at the magnetopause boundary by the anti-sunward magnetosheath flow. Magnetopause $\mathrm{KH}$ waves can cause undulation of the magnetopause and LLBL at large scales, while inducing other plasma processes at smaller scales that may allow solar wind plasma entry into the Earth's magnetosphere. The main mechanisms that allow plasma transport are proposed to be vortex-induced magnetic reconnection (VIR) (e.g. Nykyri \& Otto, 2001; Nykyri et al., 2006; Nakamura et al., 2006) and turbulence (e.g., Matsumoto \& Hoshino, 2006; Rossi, 2015). A more complex type of reconnection induced at mid-latitudes above and below the KH waves at the equatorial plane was also proposed (e.g. Faganello et al., 2012; Borgogno et al., 2015). NASA's Magnetospheric Multiscale (MMS) mission is a four-spacecraft mission (Burch et al., 2015) that enables us to study the KH waves with unprecedented high resolution down to electron scales. Direct evidence of reconnection in KH waves was reported using MMS by Eriksson et al. (2016); Eriksson et al. (2016); Li et al. (2016). Ion jets produced by VIR were observed in the compressed current sheets in the trailing (sunward facing) edges of KH waves. Evidence of other KH-induced mechanisms was also reported such as mid-latitude reconnection (Vernisse et al., 2016) and turbulence (Stawarz et al., 2016) using MMS, and kinetic Alfvén waves (Chaston et al., 2007) using Cluster.

In this work, we report observations of a Flux Transfer Event (FTE) during KH activity. An FTE is recognised in spacecraft data as a bipolar magnetic variation in the magnetopause normal direction $\left(B_{N}\right)$ with enhanced total magnetic field (e.g., Russell \& Elphic, 1978). Additionally, the total pressure, the sum of plasma and magnetic pressure, and the magnetic field strength are strongly enhanced (Paschmann et al., 1982). FTEs are proposed to be generated due to unsteady reconnection in the vicinity of single (Scholer, 1988; Southwood et al., 1988) or multiple X-line reconnection (Lee \& Fu, 1985). Small-scale FTEs have been first observed during a KH event using Time History of Events and Macroscale Interactions during Substorms (THEMIS) by 
Eriksson et al. (2009). The cross-section sizes of these FTEs were estimated to be $<0.56 R_{E}$, which are smaller than typical FTEs observed on the dayside which are of order $\sim 1 R_{E}$ (e.g. Saunders et al., 1984; Rijnbeek et al., 1984). The small-scale FTEs were found along trailing edges of KH waves detected on the dayside duskflank magnetopause during the growth phase of the waves. Eriksson et al. (2009) proposed that these small-scale FTEs were generated from unsteady reconnection in a low magnetic shear $\left(8.3^{\circ}\right)$ and low ion plasma beta $\left(\beta_{i}<0.2\right)$ environment at the KH trailing edges where current sheets might be compressed. Nevertheless, these FTEs were found to be unrelated to reconnection jets.

Magnetic islands (magnetic flux ropes or FTEs in 3-D) were independently predicted in 2-D kinetic simulations of magnetopause $\mathrm{KH}$ waves by Nakamura et al. (2011). These islands were shown to be a by-product of VIR that can be induced at multiple locations along the $\mathrm{KH}$ wave trailing edges. This is because the current sheets between $\mathrm{KH}$ vortices can be compressed down to the electron inertial length during KH evolution (Nakamura et al., 2011). The islands then propagate along the periphery of the $\mathrm{KH}$ waves with the vortical flow and merge into the $\mathrm{KH}$ vortices via secondary reconnection. This process was shown to enhance plasma mixing during the KH activity. Nakamura et al. (2013) later simulated this process using 3-D kinetic simulations and compared with observations of a KH event using THEMIS. They showed that the copious formation of magnetic islands in 2-D gives rise to magnetic flux ropes or FTEs in 3-D. In particular, the flux ropes were generated due to the tearing mode which can be induced in the compressed current sheets over a range of oblique angles (i.e., the magnetic shear angle) during the early nonlinear stage of the KH waves. Consequently, this process produces tilted flux ropes that are later incorporated into the KH vortices. The comparison with THEMIS observations shows good agreement especially for the ratio of peak-to-peak of the bipolar $B_{N}$ variation interval to the $\mathrm{KH}$ period, which is estimated to be $0.01-0.04$ while their simulation fit gives the value of 0.03. Note that the timescale of these observed VIR-induced FTEs ranges from 1 to 23 seconds (Nakamura et al., 2013), which is shorter than that of typical FTEs observed on the dayside magnetopause, which ranges from 20 seconds to 3 minutes (Kawano \& Russell, 1996; Sanny et al., 1996; Wang et al., 2005). The incorporation of the VIR-produced flux ropes into the KH vortices was shown to efficiently enhance the solar wind plasma mixing, thus they are suggested to play a role in forming the Earth's low-latitude boundary layer.

Recently, Tang et al. (2018) reported observations of an ion-scale flux rope or FTE (of 8.5 ion inertial lengths, $d_{i}$ ) during a KH event on 27 September 2016 using MMS. This FTE has a regular bipolar $B_{N}$ variation but with depressed core and total magnetic fields. The total pressure is enhanced from the background value while the plasma pressure is dominant. This FTE was therefore interpreted as a crater-type FTE (Farrugia et al., 1988; Sibeck et al., 2008) that is suggested as an early stage of a typical FTE with regular enhanced core field and dominant magnetic pressure (Zhang et al., 2010). This FTE was investigated for intense lower hybrid waves that are observed at the FTE edge and their roles in plasma transport in a crater-type FTE. Observations of ion-scale flux ropes with depressed magnetic field was also reported during the KH event on 8 September 2015 using MMS (e.g., Eriksson et al., 2016) by Sturner et al. (2018). There, they observed tripolar magnetic guide field perturbations that consist of two out-of-plane magnetic depressions during the magnetopause current sheet crossings. In particular, they observed in-plane magnetic field rotations with out-of-plane current reversal that can be interpreted as two adjacent flux ropes within a current sheet. These flux ropes were suggested to be produced from multiple reconnection. Since the flux ropes do not appear to be coalescing, whether and how these two flux ropes interact remain unknown. 
Recent MMS observations have revealed detailed structures within FTEs that were unresolved before. Øieroset et al. (2016) reported evidence of magnetic reconnection at a thin compressed current sheet between two colliding jets at the magnetic flux rope centre. Similarly, Kacem et al. (2018) reported a reconnection jet in the centre of an FTE. However, considering electron pitch angle distributions, they found that the magnetic fields on either side of the current sheet have different connectivities, indicating two interlaced flux tubes with ongoing reconnection at the interface. This work shows that what looks like an FTE does not have to be a single, homogeneous helicoidal structure as expected in a classical picture. Moreover, this illustrates in 3-D that two different sets of magnetic field lines can interact and reconnect. Øieroset et al. (2019) further showed that, at the interface between two flux tubes, magnetic flux can pile up due to the magnetic shear and this can lead to favorable conditions for reconnection.

Here we report observations of an FTE inside KH waves detected by MMS on 5 May 2017 on the post-terminator, dawn-side flank magnetopause previously analysed by Kieokaew (2019). We first describe instrumentation and event overview in Section 2. We then report observations of an FTE during the KH activity in Section 3. In particular, we report signatures that are consistent with a reconnection exhaust at the FTE current sheet in Section 4. We then discuss properties and consider magnetic topologies of this FTE in Section 5. We finally summarise and conclude in Section 6.

\section{Instrumentation and Event Overview}

We obtain data from the four-spacecraft MMS which comprise multiple instruments on board. We utilise magnetic field data from the Fluxgate Magnetometer (FGM) (Russell et al., 2016) and plasma data from the Fast Plasma Investigation (FPI) (Pollock et al., 2016). FGM instruments have a sampling time resolution down to 1 millisecond in burst mode. FPI instruments sample ions as rapidly as every 150 $\mathrm{ms}$ and electrons as rapidly as every $30 \mathrm{~ms}$. We also obtain upstream solar wind conditions from NASA's High-Resolution OMNI (HRO) database (King \& Papitashvili, 2005). Mainly data from MMS1 will be displayed.

On 5 May 2017, MMS recorded observations of quasi-periodic variations of various plasma parameters consistent with KH waves between 19:30 and 23:20 UT. Figure 1 shows orbits of the MMS spacecraft in the GSE coordinate system. MMS was located beyond the post-terminator on the dawn-side flank magnetopause as seen in Figures 1a and 1b. The average location of MMS barycentre is at $[-13.9,-18.5,3.3]$ $R_{E}$. The MMS tetrahedron size is $158 \pm 4 \mathrm{~km}$. The tetrahedron quality factor is 0.84 that is indicative of tetrahedron formation close to a regular tetrahedron and suitable for four-spacecraft technique applications (Robert et al., 1998).

Figures $2 \mathrm{a}-2 \mathrm{c}$ show the upstream solar wind conditions from HRO between 16 and 24 UT. Figure 2a shows the IMF clock angle defined as the clockwise angle of the IMF direction projected in the Y-Z plane away from the north geomagnetic pole. The IMF points mostly northward throughout the duration with an average clock angle of $10^{\circ}$. This corresponds to the average magnetic field $\mathbf{B}_{g s e}=(2.7,1.2,6.6) \mathrm{nT}$. The average flow velocity in Figure $2 \mathrm{c}$ is $|\mathbf{V}|=380 \mathrm{~km} \mathrm{~s}^{-1}$. Other parameters (not shown) include average Alfvén Mach number, $M_{A}=4.8$, ion beta, $\beta_{i}=0.4$, ion density, $n_{i}=3.8 \mathrm{~cm}^{-3}$ and solar wind dynamic pressure, $P_{d y n}=1.1 \mathrm{nPa}$.

Figures 2d - 2k show observations from MMS1. Ion and electron energy-time spectrograms show variable fluctuations but overall decreasing high energy particle fluxes in Figures 2d and 2e, respectively. These indicate that MMS1 was transiting from the magnetosphere to the magnetosheath. The magnetospheric plasma (from 16:00 to 17:05 UT) is characterised by very low flow speed in Figure 2i, low ion density 
MMS Location for 2017-05-05 20:00:00 UTC

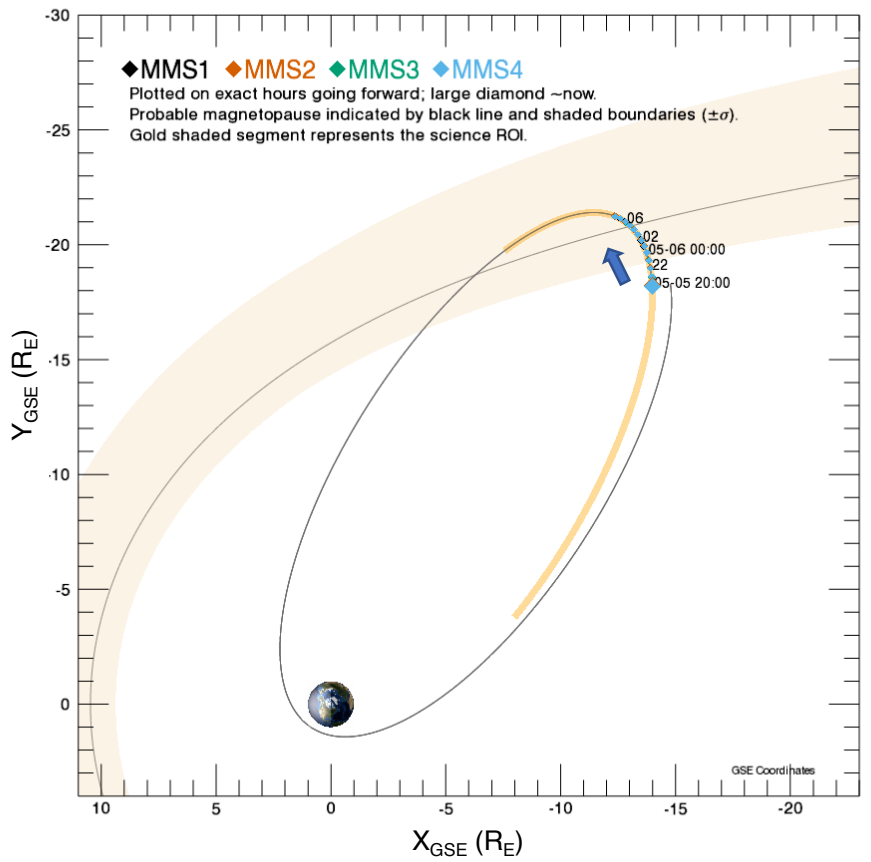

MMS Location for 2017-05-05 20:00:00 UTC

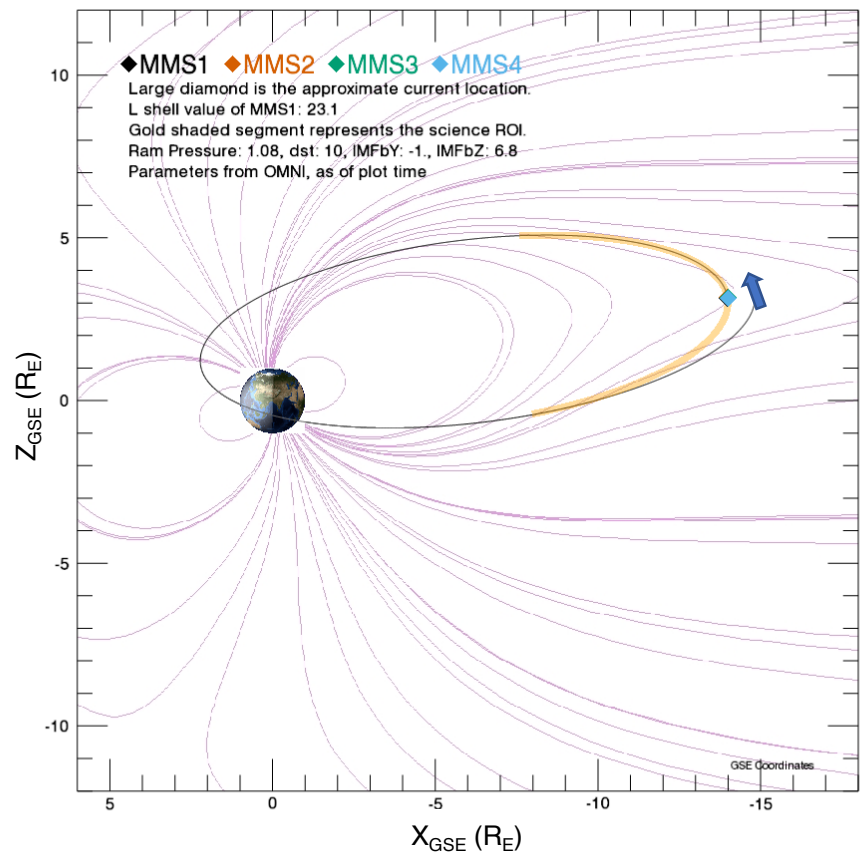

Figure 1. MMS orbit on 5 May 2017 at 20:00 UT shown in the Geocentric Solar Ecliptic (GSE) coordinates in $X-Y$ (top) and $X-Z$ (bottom) projections, respectively. 


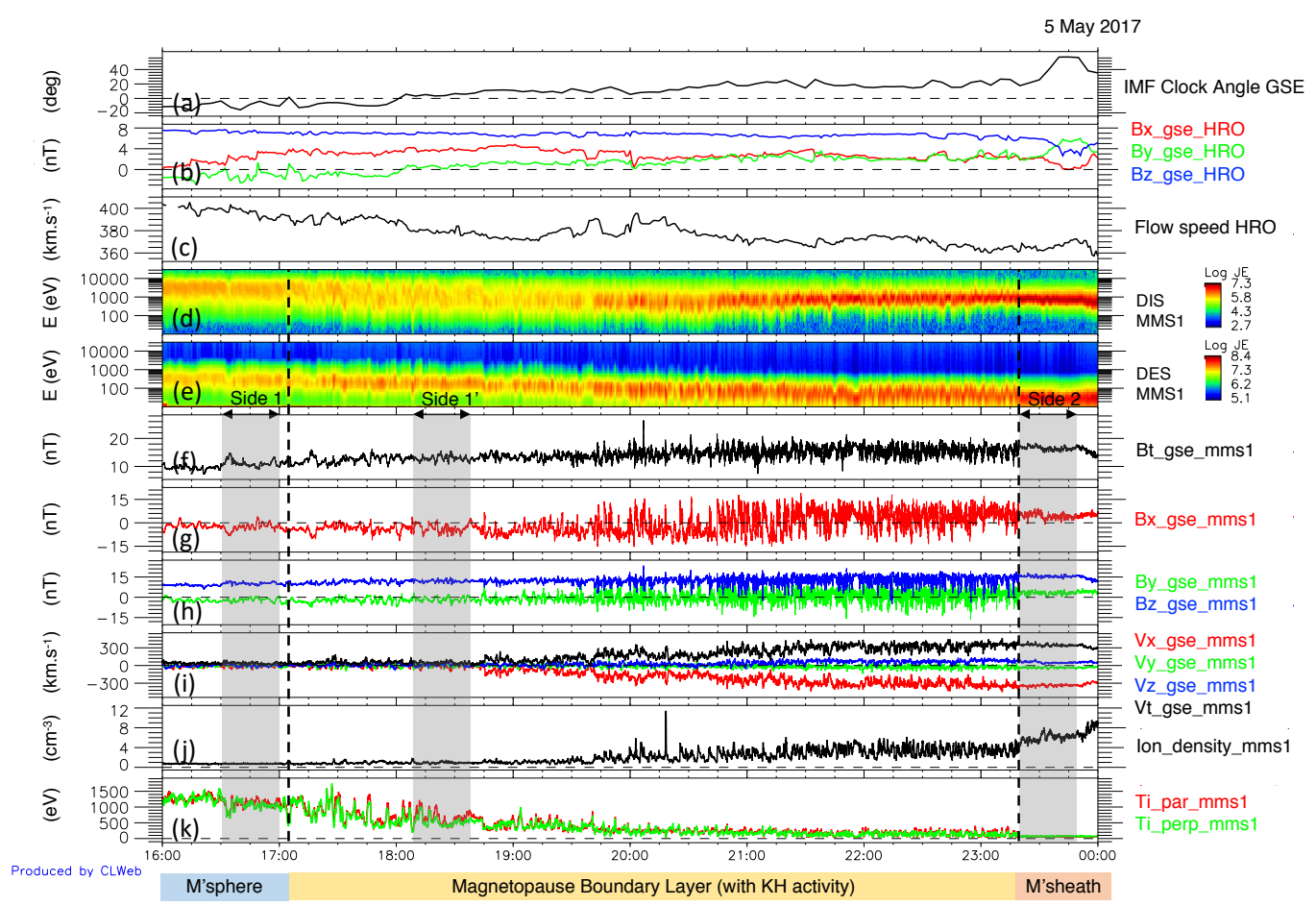

Figure 2. Overview of the 5 May 2017 event between 16 UT and 24 UT from HRO (panels (a) - (c)) and MMS1 (panels (d) - (k)): (a) IMF clock angle, (b) upstream magnetic fields, (c) upstream ion flow speed, (d) ion energy-time spectrogram, (e) electron energy-time spectrogram, (f) magnetic field strength $B_{t}$, (g) magnetic field component $B_{x}$, (h) magnetic field components $B_{y}$ and $B_{z}$, (i) ion bulk velocity, (j) ion number density, and (k) ion temperature. The boundary layer interval is marked between the two vertical dashed lines. Shaded areas mark the regions for the $\mathrm{KH}$ onset calculation (see text).

in Figure 2j, and high ion temperature in Figure 2k. The magnetosheath plasma (from 23:20 UT to 24 UT) is characterised by faster flow, higher ion density, and lower ion temperature. Figure $2 \mathrm{f}$ shows that the magnetic field strength $B_{t}$ is variable but gradually increases from $\sim 10 \mathrm{nT}$ in the magnetosphere to $\sim 16 \mathrm{nT}$ in the magnetosheath. The increasing magnetic field strength is mainly contributed by the magnetic field component $B_{x}$ and $B_{z}$ as shown in Figures $2 \mathrm{~g}$ and $2 \mathrm{~h}$. The interval between the two vertical dashed lines may be identified as the boundary layer.

To test whether the boundary conditions satisfy the $\mathrm{KH}$ onset condition, we apply the linear theory (Chandrasekhar, 1961) to obtain a KH growth rate. Using an assumption that the main $\mathrm{KH}$ perturbation is in $y$ direction and the plasma flow velocity $\mathbf{U}$ and the magnetic field $\mathbf{B}$ have only $x$ and $z$ components (e.g. Nakamura et al., 2006), the onset condition can be written as

$$
\begin{aligned}
\left(\frac{\gamma}{k}\right)^{2}= & \frac{\rho_{1} \rho_{2}}{\left(\rho_{1}+\rho_{2}\right)^{2}}\left(\Delta U_{x} \cos \theta+\Delta U_{z} \sin \theta\right)^{2} \\
& -\left[\left(B_{1 x} \cos \theta+B_{1 z} \sin \theta\right)^{2}+\left(B_{2 x} \cos \theta+B_{2 z} \sin \theta\right)^{2}\right] /\left[\mu_{0}\left(\rho_{1}+\rho_{2}\right)\right]
\end{aligned}
$$

where indices 1,2 are for the magnetosphere and magnetosheath, respectively, $\rho$ is the plasma mass density, $\mathbf{k}=(k \cos \theta, 0, k \sin \theta)$ is the wave propagation direction with the wave number $k=2 \pi / \lambda$, and $\mu_{0}$ is the vacuum permeability. A positive growth rate, $\gamma / k>0$, indicates that the $\mathrm{KH}$ onset condition is satisfied. This growth rate is 
calculated in magnetopause boundary coordinates where $y$ is normal and outward from the unperturbed magnetopause, $z$ is along the geomagnetic axis, and $x$ completes the orthogonal system. Here the angle $\theta$ is defined between $\mathbf{k}$ and $x y$-plane of the shear flow. Using the magnetopause model from Shue et al. (1997), the transformation for this coordinate system calculated at $20 \mathrm{UT}$ (the middle time between 16 and $24 \mathrm{UT})$ is found to be $\mathbf{x}=[-0.990,-0.134,0.047], \mathbf{y}=[0.141,-0.975,0.170]$, and $\mathbf{z}=[0.024,0.168,0.985]$ in the GSE coordinates.

We obtain the magnetospheric values (side 1) for the interval between 16:30 and 17:00 UT and the magnetosheath values (side 2) for the interval between 23:20 and 23:50 UT as shaded in Figure 2. The average plasma number densities are found to be $n_{1}=0.7 \pm 0.1 \mathrm{~cm}^{-3}$ and $n_{2}=6.0 \pm 0.7 \mathrm{~cm}^{-3}$. The average plasma velocities are $\mathbf{U}_{1}=$ $(-2.7,-5.3,6.1) \mathrm{km} \mathrm{s}^{-1}$ and $\mathbf{U}_{2}=(-349.3,0.6,73.6) \mathrm{km} \mathrm{s}^{-1}$, giving $\Delta \mathbf{U}=\mathbf{U}_{1}-\mathbf{U}_{2}=$ $(346.6,-5.9,-67.5) \mathrm{km} \mathrm{s}^{-1}$. The average magnetic fields are $\mathbf{B}_{1}=(-2.9,3.2,9.9) \mathrm{nT}$ and $\mathbf{B}_{2}=(4.7,0.2,15.7) \mathrm{nT}$. Application of the formula (1) with an arbitrary angle $\theta$ yields a positive and maximum growth rate of $\gamma / k=101 \mathrm{~km} \mathrm{~s}^{-1}$ at $\theta_{0}=-11.5^{\circ}$. This shows that the shear flow conditions indeed fulfill the $\mathrm{KH}$ criterion.

In some observations, while the $\mathrm{KH}$ waves are evidenced, the $\mathrm{KH}$ onset condition is not satisfied using the shear flow conditions on either side of the magnetopause. It was suggested that the inner edge of the boundary layer may be more susceptible to the $\mathrm{KH}$ mode due to the absence of magnetic shear which suppresses the instability (e.g., Sckopke et al., 1981; Ogilvie \& Fitzenreiter, 1989; Farrugia et al., 1998; Farrugia et al., 2000). To ascertain this point, we may calculate the $\mathrm{KH}$ growth rate given that the side 1' represents an inner boundary interval as follows. The inner edge interval may be identified between 18:10 and 18:40 UT as shaded in Figure 2 where the KH instability is still absent but is close to the $\mathrm{KH}$ activity. In that case, we obtain the average plasma number density $n_{1}^{\prime}=0.91 \pm 0.15 \mathrm{~cm}^{-3}$, plasma velocity $\mathbf{U}_{1}^{\prime}=(-6.5,-3.4,15.1) \mathrm{km}$ $\mathrm{s}^{-1}$, and magnetic field $\mathbf{B}_{1}^{\prime}=(-3.5,3.1,11.6) \mathrm{nT}$. Using these values, we obtain a maximum growth rate of $108 \mathrm{~km} \mathrm{~s}^{-1}$ at $\theta_{0}=-5.7^{\circ}$. Therefore, the maximum growth rate $\gamma / k$ should be in range $101-108 \mathrm{~km} \mathrm{~s}^{-1}$ and it confirms that this event is very likely due to the $\mathrm{KH}$ instability.

\section{Observations of a Flux Transfer Event in a $\mathrm{KH}$ wave}

Figure 3 shows MMS1 observations between 20:00 UT and 20:15 UT. Panels (a - e) show magnetic fields, ion number density, ion temperature, ion bulk velocity, and ion pressure respectively. The magnetic fields show large-scale $B_{Y}$ and $B_{X}$ variations that correspond to the large-scale vortical/wavy structure of the KH waves. The ion temperature and density show more or less periodic transitions from the magnetospheric-like (hot and tenuous) to magnetosheath-like (cold and dense) sides as marked by vertical dashed lines. Each of these transitions is consistent with an outbound crossing of $\mathrm{KH}$-perturbed magnetopause that is known as a $\mathrm{KH}$ wave trailing edge or the sunward facing edge (e.g. Otto \& Fairfield, 2000).

In Figure 3a, a clear bipolar variation can be seen in the $B_{Y}$ component (green) with an enhancement in the magnetic field magnitude (black) near the third vertical dashed line, as marked by a purple arrow. At the same time in Figure 3e, the total pressure (black) is strongly enhanced to twice the ambient value. This enhanced total pressure is mainly due to an enhancement in magnetic pressure (red). We interpret these signatures as a passage of an FTE-type structure. This structure, while embedded in the large-scale boundary layer crossing, is distinct from other current sheet crossings in terms of the local properties such that there is no sharp plasma density and temperature variations, i.e., compared to those marked by the vertical dashed lines. Furthermore, the magnetic field amplitude and its $B_{Y}$ variation are much larger than 
typical compressed current sheets in $\mathrm{KH}$ waves (the event stands-out as very peculiar in that respect when one looks at all MMS KH events).

At the flank magnetopause, the bipolar $B_{Y}$ variation of an FTE crossing may resemble the passage of a rolled-up KH vortex (Nykyri et al., 2003). However, an FTE passage can be easily distinguished because its total pressure must reach a local maximum, while for a $\mathrm{KH}$ vortex passage the total pressure typically reaches a local minimum (e.g. Otto \& Fairfield, 2000). Also, an FTE signature would appear isolated or separated by long periods of quiet activity (Russell et al., 1996), while the KH waves appear more or less periodically. Moreover, the $B_{Y}$ (and $B_{X}$ ) variations due to the $\mathrm{KH}$ waves tend to be longer with relatively smooth transitions on large scale. The identified FTE-type structure clearly appears as a very localised additional structure within the overall larger-scale $B_{Y}$ variation from the $\mathrm{KH}$ waves.

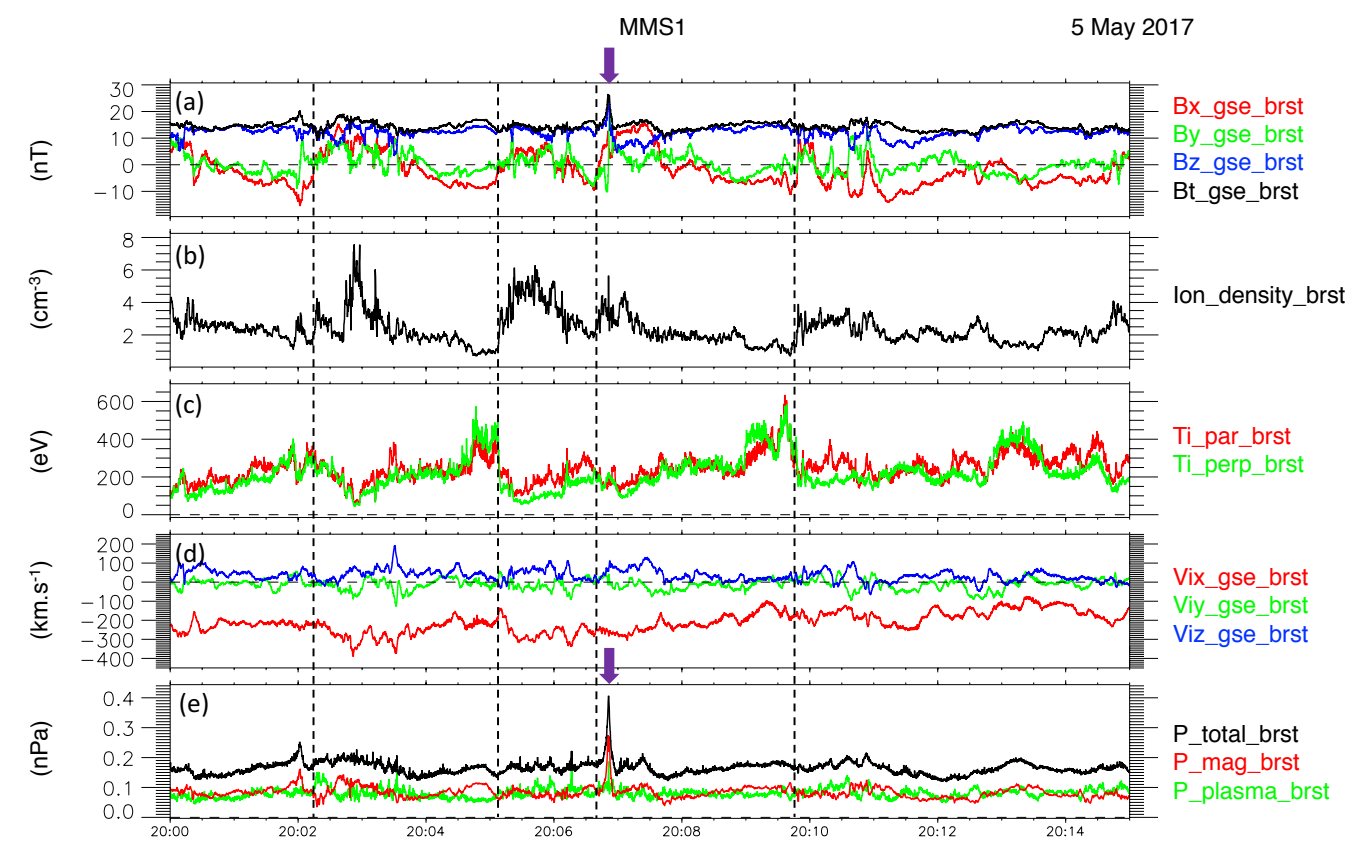

Figure 3. MMS1 observation of a FTE during the KH wave activity between 20:00 UT and 20:15 UT on 5 May 2017. (a) magnetic fields in GSE coordinates, (b) ion number density, (c) ion temperature, (d) ion bulk velocity in GSE coordinates, and (e) total pressure (black), magnetic pressure (red), and plasma pressure (green). Vertical dashed lines roughly mark transitions from the magnetospheric to magnetosheath plasma which are outbound magnetopause crossings and correspond to $\mathrm{KH}$ wave trailing edges (sunward facing edges). Purple arrows indicate an FTE passage at 20:06:50 UT. The FTE is observed near the KH trailing edge characterised by $B_{x}$ transition from negative to positive in panel (a).

To characterise the configurations of the current sheets, we perform a boundary normal analysis as follows. Application of the hybrid minimum variance analysis (MVA) (Gosling \& Phan, 2013) (see the supplementary information) yields normal directions in GSE as $[0.08,-0.98,-0.18],[0.33,-0.93,0.17],[0.21,-0.90,-0.39]$, and $[0.29,-0.95,0.11]$, for the marked current sheets from left to right in Figure 3; while the normal of the FTE current sheet is $[0.93,0.12,-0.35]$ (for full details see the sup- 
plementary Table S1). In other words, the normal of the marked current sheets are mostly in $-Y_{\text {gse }}$ (outward from the dawn flank) while that of the FTE current sheet is in $+X_{g s e}$ (sunward), which is rather orthogonal. Application of the regular MVA method (Sonnerup \& Cahill, 1967) also yields consistent results (see Table S1). Hence, the normal direction of the FTE current sheet is rather unique, indicating a distinct structure unlike the KH-perturbed magnetopause current sheets.

Figure 4 shows the passage of the isolated FTE at MMS1 between 20:06:41 UT and 20:07:01 UT (top, panels $(\mathrm{a}-\mathrm{j})$ ) and its zoom-in (bottom, panels $(\mathrm{k}-\mathrm{o})$ ) between 20:06:46 UT and 20:06:56 UT. Note that an extension of this Figure to include the magnetopause current sheet prior to the FTE can be found in the supplementary Figure S1. In Figure $4 \mathrm{a}$, a clear reversal of the magnetic field $B_{y}$ (green) from negative to positive can be seen around 20:06:51.2 UT marked by the time $t_{c}$, regarded here to mark the FTE centre. Around the FTE centre, we note enhancements of magnetic field strength $B_{t}$ (black) and notably the northward component $B_{z}$ (blue). The magnetic field is also shown in the magnetopause boundary LMN coordinates similar to those in Section 2 but with conventional notations for displaying an FTE (Russell \& Elphic, 1979) in Figure 4k. In this coordinate system, $\mathbf{l}=\mathbf{z}, \mathbf{m}=-\mathbf{x}$ and $\mathbf{n}=-\mathbf{y}$ in Section 2.

At the time $t_{c}$ marking the FTE centre, the ion number density is increased in Figure $4 b$, the electron pitch angle distribution (ePAD) in the energy range $(181-542$ $\mathrm{eV}$ ) shows counter-streaming electrons in Figure 4d, and the electron temperature features strong parallel heating in Figure 4f. Around the FTE centre, between $t_{1}$ and $t_{2}$, the total pressure shown as a black line in Figure $4 \mathrm{~g}$ is enhanced up to twice the ambient total pressure $(\Delta P=0.2 \mathrm{nPa})$, consistent with Paschmann et al. (1982). This total pressure enhancement is dominated by the magnetic pressure (red) as expected for a magnetic flux rope. The ion $\beta$ in Figure $4 \mathrm{j}$ shows a decreased value down to 0.5 surrounding the FTE centre. The magnetic pressure enhancement dips to balance the spike in plasma pressure (green) at time $t_{c}$, so that the total pressure varies relatively slowly. The current density in Figure 4h, measured by the four-spacecraft curlometer technique (Dunlop et al., 1988), shows an increase in $J_{z}>0$ (blue) surrounding the FTE centre. The current density is also shown in the magnetopause boundary LMN coordinates in Figure $4 \mathrm{l}$ which shows the strong positive $J_{l}$ (blue) around the FTE centre. This out-of-plane current sustains the in-plane magnetic field rotation as seen in Figure 4a. In Figure 4i, there is a variation in ion bulk velocity component $V_{y}$ (green) from negative to positive close to $t_{c}$. Despite being small in magnitude, this $V_{y}$ change corresponds to the bipolar $B_{y}$ variation. Note that this jet is observed inside the magnetopause, as the plasma on both sides of the jet is boundary layer-type plasma (see also Figure 3d). We will investigate this feature in the next section.

We now analyse geometrical structures of the FTE. Here we apply magnetic curvature analysis (MCA), a four-spacecraft technique developed by Shen et al. (2003). The MCA technique calculates magnetic curvature $\mathbf{b} \cdot \nabla \mathbf{b}$, where $\mathbf{b}=\mathbf{B} /|\mathbf{B}|$, that is proportional to the perpendicular component of magnetic tension $\left(\mathbf{B} \cdot \nabla \mathbf{B} / \mu_{0}\right)$. The technique yields curvature vector $\mathbf{C}$ and curvature radius $R_{c}=1 /|\mathbf{C}|$ that can be used to characterise geometrical properties of magnetic structures. Magnetic curvature has been resolved in Cluster observations of various plasma structures such as current sheets (Shen et al., 2003), magnetic reconnection (Runov et al., 2003, 2005), magnetic flux ropes (Yang et al., 2014) and MMS observations of electron diffusion regions (Lavraud et al., 2016). It has also been applied to investigate KH wave structures in MHD simulations (Kieokaew et al., 2018) and Cluster observations (Kieokaew \& Foullon, 2019).

Figures $4 \mathrm{~m}$ - 4o show results from the MCA technique. Figure $4 \mathrm{~m}$ shows magnetic curvature components; the component $C_{m}$ (red) is dominant, indicating that the magnetic fields are mainly curved in the $\pm \mathbf{m}$ direction (sunward or tailward direction). A transition from $C_{m}<0$ (sunward) to $C_{m}>0$ (tailward) which is near to the FTE 


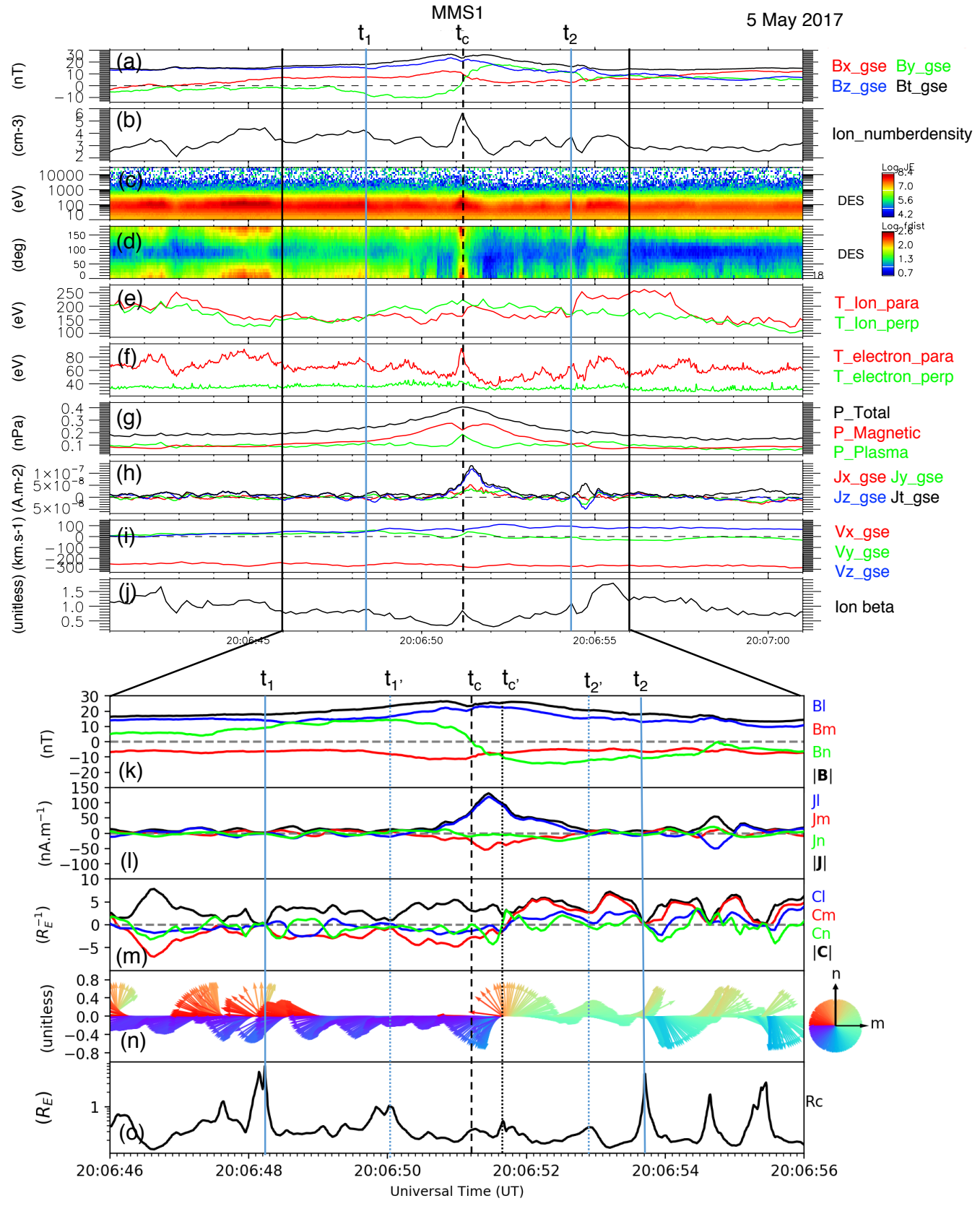

Figure 4. (top) Overview of MMS1 measurements during the FTE crossing on 5 May 2017 between 20:06:41 and 20:07:01 UT in GSE coordinates: (a) magnetic fields; (b) ion number density; (c) electron energy spectrogram; (d) electron pitch angle distribution (ePAD) for electron energy range 181 - $542 \mathrm{eV}$; (e and f) parallel (red) and perpendicular (green) ion and electron temperatures; (g) total (black), magnetic (red), and plasma (green) pressures; (h) current density; (i) ion bulk velocity and (j) ion plasma $\beta$. (bottom) A zoom-in of the top panels during 20:06:46 UT and 20:06:56 UT in the magnetopause boundary LMN coordinates: (k) magnetic fields; (l) current density; (m) curvature vector components; (n) 2-D normalised curvature projection in the $M-N$ plane; and (o) radius of curvature, $R_{c}$. Vertical lines mark $t_{c}$ (FTE centre) at the centre of the bipolar $B_{Y}$ variation, $t_{1}$ and $t_{2}$ when $R_{c}$ reaches its maxima, $t_{c}^{\prime}$ (FTE core) when all curvature components turn from negative to positive values, and $t_{1}^{\prime}$ and $t_{2}^{\prime}$ when $R_{c}$ reaches the local maxima near the FTE core, whigh mark the boundary of the flux rope. 
centre $\left(t_{c}\right)$ is marked at time $t_{c^{\prime}}$. Figure $4 \mathrm{n}$ shows a time series of the $2 \mathrm{D}$ normalised curvature vector in the $M-N$ plane, $C_{m n}=\left(C_{m} \mathbf{m}+C_{n} \mathbf{n}\right) / \sqrt{C_{m}^{2}+C_{n}^{2}}$ with angle colour-coded by $\arctan \left(C_{n} / C_{m}\right)$. One can see a clear turning of the curvature direction from $-\mathbf{m}$ (sunward) to $+\mathbf{m}$ (tailward) at $t_{c^{\prime}}$, interpreted as the FTE core. This behaviour of the curvature is indicative of a cylinder-like magnetic structure moving over the spacecraft in the cross-section wise direction. The radius of curvature $\left(R_{c}\right)$ in Figure 4o typically reaches a local maximum when $|\mathbf{C}|$ tends to 0 , meaning that the magnetic field has no curvature and is therefore straight, e.g., at $t_{1}$ and $t_{2}$. Between $t_{1}$ and $t_{2}$, we can recognise that curvature radius reach local maxima in the core of the FTE at $t_{c}$ and $t_{c}^{\prime}$ and in the surrounding at $t_{1}^{\prime}$ and $t_{2}^{\prime}$. We interpret the interval $t_{1}^{\prime}$ to $t_{2}^{\prime}$ as the inner part of the FTE. The minimum $R_{c}$ in this interval is $0.17 R_{E}$ or $1080 \mathrm{~km}$. This value can be used to set an upper limit for the FTE cross-section at about 2160 $\mathrm{km}$, which is $17.6 d_{i}$. This is within the extent of the FTE crossing of $\sim 2,500 \mathrm{~km}$ estimated using the average ion bulk speed between 20:06:46 UT and 20:06:56 UT of $\sim 250 \mathrm{~km} . \mathrm{s}^{-1}$. The $R_{c}$ at the FTE core is $0.5 R_{E}$ which is the local maximum and it is decreasing away from the FTE core. The large $R_{c}$ at the centre and smaller value away from the core were also found by Yang et al. (2014). The curvature radius and curvature vector profiles imply that our FTE structure is consistent with a helicoidal flux rope model (e.g., Bothmer \& Schwenn, 1998).

To check whether this FTE is consistent with the magnetic islands (flux ropes, FTEs) produced by vortex-induced reconnection, we compare the size of this flux rope to those predicted in simulations by Nakamura et al. (2011, 2013). Nakamura et al. (2013) obtain a simulation fit for the ratio of peak-to-peak distance of the bipolar $B_{y}$ variation to the $\mathrm{KH}$ wavelength to be 0.03 . This value is in agreement with observations from their KH event detected by THEMIS which gives a range of the ratio of peak-topeak time interval of the bipolar $B_{Y}$ signature, $\Delta T_{\text {peak }}$, to the $\mathrm{KH}$ period, $\Delta T_{\mathrm{KH}}$, to be $0.01-0.04$. For our FTE, we measure $\Delta T_{\text {peak }}$ to be about 2.4 s. Using $\Delta T_{\mathrm{KH}}=93 \pm 35$ $\mathrm{s}$, obtained from averaging the time intervals between outbound crossings in Figure 3 , we obtain the ratio $\Delta T_{\text {peak }} / \Delta T_{\mathrm{KH}}$ to be $0.02-0.04$ that is in excellent agreement with Nakamura et al. (2013). Therefore, this FTE is consistent with a vortex-induced flux rope.

\section{Observations of reconnection inside the FTE}

Figure 5 shows observations between 20:06:46 and 20:06:56 UT from all four MMS spacecraft. Here we utilise the local current sheet LMN coordinates. In this coordinate system, $\mathbf{L}$ directs along the reconnecting component (approximately $+Y_{g s e}$ ), $\mathbf{M}$ is along the guide field direction which is about northward $\left(+Z_{g s e}\right)$, and $\mathbf{N}$ completes the right-hand orthogonal system. Since the MMS2 (orange) current sheet crossing is observed in the middle of all crossings, we obtain the LMN transformation using the hybrid MVA method (Gosling \& Phan, 2013) at MMS2 and then apply the same to other spacecraft data. The current sheet normal $N$ is obtained from the cross product of the 1-second averaged magnetic fields before entering and after exiting the current sheet; the sign of $N$ is chosen such that it is directed away from Earth. A first maximum variance direction $L_{1}$ is obtained from the MVA. The $M$ component is then computed from $N \times L_{1}$. Finally, $L$ is calculated from $M \times N$ so that the local current sheet LMN coordinates are orthonormal. The transformation is found to be $L_{G S E}=$ $[-0.192,0.968,-0.162], M_{G S E}=[0.423,0.23,0.876], N_{G S E}=[0.885,0.1,-0.454]$.

Figures $5 \mathrm{a}$ and $5 \mathrm{~b}$ show magnetic field components $B_{L}$ and $B_{M}$, respectively. A bipolar variation is seen in the magnetic field component $B_{L}$. It changes from the average value (from four MMS spacecraft) of $-9.5 \mathrm{nT}$ to $8.1 \mathrm{nT}$, which is nearly symmetric. There is an enhancement in the magnetic field component $B_{M}$ (e.g., guidefield component) by about $10 \mathrm{nT}$, from the background value of $15 \mathrm{nT}$ to the enhanced value of $25 \mathrm{nT}$ at the current sheet centre. One can notice a small bipolar perturbation 


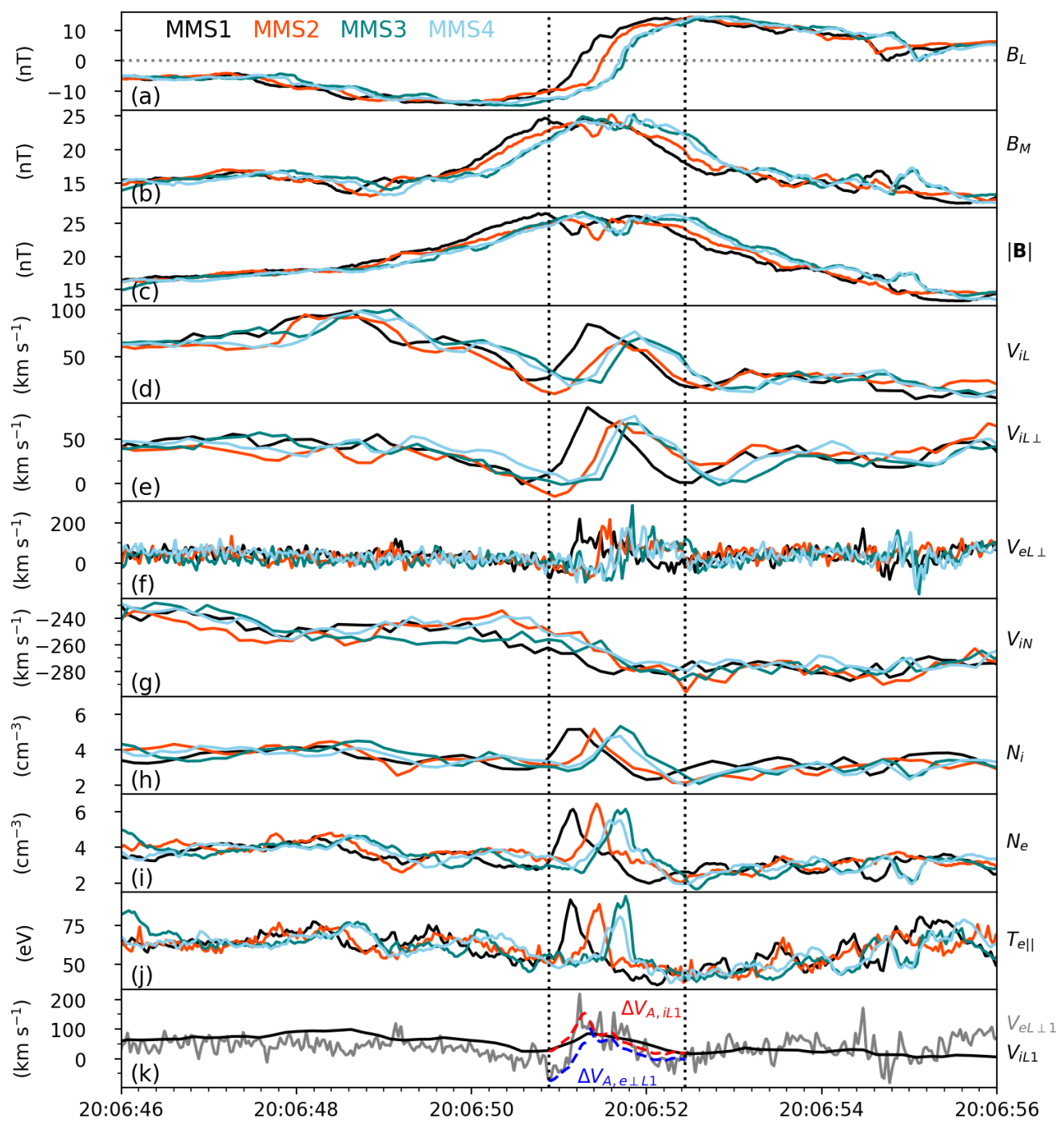

Figure 5. (a-i) Four-spacecraft crossing of the FTE current sheet between 20:06:46 and 20:06:56 UT in the local current sheet LMN coordinates. (a and b) Magnetic field components $B_{L}$ and $B_{N}$, respectively; (c) magnetic field strength $|\mathbf{B}|$; (d) ion velocity component $V_{i L}$; (e and f) ion and electron perpendicular velocity components $V_{i L \perp}$ and $V_{e L \perp}$, respectively; (g) ion velocity component $V_{i N}$; (h and i) ion and electron number densities $N_{i}$ and $N_{e}$, respectively; (j) electron parallel heating $T_{e \|}$; and (k) the $L$ component of ion velocity $V_{i L 1}$ and electron perpendicular velocity $V_{e L \perp 1}$ at MMS1 with the $L$ component of the predicted velocity from the Walén relation (Alfvénic flows) using as reference on either side of the current sheet for the ion (red dashed line) and electron (blue dashed line) velocities. Vertical dotted lines mark the interval of the ion jet (panel (d)) seen by MMS1 (black). This ion jet is visible at all spacecraft but with some relative delays. 
of the $B_{M}$ component at around 20:06:51.4 UT that is best seen at MMS2 (orange). The peak-to-peak value of this $B_{M}$ perturbation is around $2.6 \mathrm{nT}$. This perturbation may be consistent with the Hall magnetic field pattern (e.g. Sonnerup, 1979) which creates a quadrupolar magnetic field perturbation on the guide magnetic field as seen from above a reconnection X-line; this pattern would be seen as a bipolar perturbation for a spacecraft crossing on one side of a reconnection exhaust. However, we note that this perturbation is small and less clear at other spacecraft.

In addition, the asymptotic guide field $B_{M}$ magnitude is around 1.5 times of the reconnecting component $B_{L}$ magnitude. This large guide field may lead to asymmetries of the reconnection exhaust (e.g. Eastwood et al., 2010; Øieroset et al., 2016). The guide-field effect is noticeable, i.e., in the ion density which is shifted to one side in Figure 5h. Figure 5c shows the magnetic field strength $|\mathbf{B}|$ which is generally enhanced at the current sheet, but a dip near the centre for about $3 \mathrm{nT}$ is observed at every spacecraft. This dip is consistent with a conversion of the magnetic energy to kinetic energy at the reconnecting current sheet.

An ion jet is visible in the velocity component $V_{i L}$ shown in Figure 5d, which appears around the current sheet centre $\left(t_{c}\right)$ marked between the black vertical dotted lines for MMS1. The magnitude of this jet is $\Delta V_{i L 1} \sim 61 \mathrm{~km} \mathrm{~s}^{-1}$. This ion jet is mainly contributed by the perpendicular component (to the magnetic field) of the bulk velocity as seen in Figure 5e. It is also visible for the perpendicular component of electrons, which shows the jet speed of $\sim 250 \mathrm{~km} \mathrm{~s}^{-1}$ in Figure $5 \mathrm{f}$. To test whether this jet is consistent with a reconnection jet, we perform a Walén test (e.g. Hudson, 1970; Paschmann et al., 1986) on the ion and electron jet interval in Figure 5k. The magnetic field component $B_{L}$ and ion velocity component $V_{i L}$ have a positive correlation before the ion jet peak time and a negative correlation after it, consistent with a reconnection jet. In Figure $5 \mathrm{~g}$, the ion velocity component $V_{i N}$ shows a change from around -250 $\mathrm{km} \mathrm{s}^{-1}$ before the current sheet to around $-280 \mathrm{~km} \mathrm{~s}^{-1}$ after the current sheet crossing. This velocity change in the normal direction is consistent with converging flows when crossing from $B_{L}<0$ (carried by the positive jet) to $B_{L}>0$ (carried by the negative jet).

For a rotational discontinuity, the ion jet speed can be predicted using the relation $\Delta \mathbf{V}_{\mathbf{A}} \sim \pm \Delta \mathbf{B} /\left(\mu_{0} m_{p} N_{i}\right)^{1 / 2}$ (Phan et al., 2004; Gosling et al., 2005) where the positive and negative signs are applied for the positive and negative correlations between $\mathbf{B}$ and $\mathbf{V}$, respectively. We apply the Walén relation for the $L$ component of the magnetic field to obtain the Alfvénic jet speed in $L$ direction for MMS1 in Figure 5k. The predicted jet speed is shown as a red dashed line $\left(\Delta V_{A, i L 1}\right)$ and a blue dashed line $\left(\Delta V_{A, e \perp L 1}\right)$ using the ion (black) and electron (grey) velocities on either side of the current sheet as reference, respectively. The predicted jet speed shows an overall agreement with the ion velocity. However, the predicted jet speed is around $109-134 \mathrm{~km} \mathrm{~s}^{-1}$, while the observed ion speed is only $61 \mathrm{~km} \mathrm{~s}^{-1}$. Therefore, the observed ion jet speed is about $50 \%$ of the predicted jet speed. The ion jet speed is slowest at MMS3 as seen in Figure $5 \mathrm{~d}$ with the value of $45 \mathrm{~km} \mathrm{~s}^{-1}$ or $37 \%$ of the Alfvén speed. Such a low observed value may be due to the proximity to the X-line (Phan et al., 2016), possibly because the jet is not yet fully developed spatially at the MMS location. We may also note that the electron jet is much faster and becomes super-Alfvénic near the current sheet centre as seen in Figure 5k. A super Alfvénic electron jet was also found in the middle of reconnecting current sheet close to the X-line by Phan et al. (2007). To support the above argument, we determine the distance of the crossing from the X-line as follows.

We may estimate the distance from the X-line using the assumption that a ratio of the thickness to length of a reconnecting current sheet is 0.1 , that is, the opening angle of the reconnection exhaust is the same as the angle from the canonical aspect ratio of the diffusion region (e.g. Phan et al., 2016; Lavraud et al., 2016). To determine the thickness, we first obtain the crossing time of the exhaust. Indeed, there is some 
ambiguity in the determination of the extent of the reconnection exhaust. By comparison with simulation, Øieroset et al. (2016) determine the first and second separatrices of the exhaust where the density starts to change near the ion jet boundary. We mark the extent of an ion jet seen by MMS1 (black) as bounded by vertical dashed lines in Figure 5. Using all four MMS spacecraft, the average crossing time interval is found to be $1.8 \pm 0.2 \mathrm{~s}$. Application of multispacecraft timing analysis (e.g. Russell et al., 1983 ) on the magnetic field component $B_{y}$ for the interval of current sheet crossing yields a normal speed of $240 \mathrm{~km} \mathrm{~s}^{-1}$ at MMS1. The ion inertial length $\left(d_{i}\right)$ is found to be $134 \pm 5 \mathrm{~km}$ using the averaged ion density of $2.9 \pm 0.2 \mathrm{~cm}^{-3}$ at the exhaust boundaries determined at the four spacecraft. The current sheet thickness is therefore $3.2 \pm 0.5 d_{i}$. This gives an averaged distance from the X-line of $16 \pm 4 d_{i}$. Following the same process, we found that MMS3 is closest to the X-line with a distance of 11 $d_{i}$, while it observes the slowest jet speed. Thus, it is likely that the ion jet is not fully developed near the X-line. There are also other possibilities that may account for a sub-Alfvénic ion jet such as a large plasma- $\beta$ (Paschmann et al., 1986; Phan et al., 1996), the presence of slow shocks (Øieroset et al., 2000), or an ion temperature anisotropy (Haggerty et al., 2018). In conclusion, there is a jet at the current sheet along the $L$ component, as expected for reconnection albeit with a lower amplitude.

There is also other evidence that supports the interpretation of a reconnection exhaust. In Figures $5 \mathrm{~h}$ and 5i, the ion and electron number densities become enhanced for about $3 \mathrm{~cm}^{-3}$ around the current sheet centre, consistent with the exhaust region being populated by the mixing of plasmas from either side of the boundary for symmetric reconnection (Gosling et al., 2005). There is a strong parallel electron heating of about $50 \mathrm{eV}$ that is also a typical signature of reconnection in Figure 5j. These signatures, along with the observed jet (Figures 5d - 5f), are consistent with a reconnection exhaust, and all of them are observed at all four spacecraft. These signatures are embedded in between the converging flows in the current sheet normal direction as seen in Figure 5g.

It is worth noting that our reported exhaust has a configuration unlike that of a VIR-exhaust. For a type-I VIR exhaust, we expect the jet to be directed along a KH wave trailing edge, e.g., as shown in Figure 4 of Eriksson et al. (2016) (a similar sketch is shown here in Figure 7a). From the analysis in the Section 3, we found that the averaged normal of current sheets associated with the $\mathrm{KH}$ wave trailing edges is $[0.23,-0.94,0.02]_{G S E}$ based on the hybrid MVA method. This implies that the VIR jet direction should be more or less perpendicular to this normal direction in $-Y_{G S E}$, which is $\pm X_{G S E}$. However, we found that the direction of the jet at the FTE central current sheet is $L_{F T E-C S}=[-0.18,0.97,-0.15]_{G S E} \sim Y_{G S E}$, which is not the expected direction of the VIR jet. We will discuss a plausible mechanism for the production of this jet in the middle of the FTE in the next section.

\section{Discussion}

We report the observation of an FTE at the trailing edge of a $\mathrm{KH}$ wave using MMS. This FTE structure is characterised by a bipolar $B_{Y}$ variation along with the other properties that are embedded within the large-scale magnetic field and plasma variations associated with the $\mathrm{KH}$ vortical/wavy structures. While compressed magnetopause current sheets can also lead to a bipolar $B_{Y}$ variation, our reported structure is distinct in terms of local properties on the basis of its orientation (jet direction and normal of the current sheet with respect to regular VIR jets and normals of compressed current sheets in KH waves), as well as because such that there is no plasma density and temperature transitions and velocity shear (as commonly found for the magnetopause). In fact, the jet in the FTE central current sheet is observed between two plasma regions of boundary-layer-type (i.e., inside the magnetopause). Because nei- 
ther side of the reconnecting current sheet is the magnetosheath proper, this boundary is not the magnetopause, by definition.

It was shown in simulations by Nakamura et al. $(2011,2013)$ that the smallscale FTEs in KH waves are generated due to VIR at multiple locations along the compressed current sheet (i.e., the trailing edge) between $\mathrm{KH}$ vortices. This process is analogous to the large-scale multiple X-line model generation of an FTE (e.g. Lee \& Fu, 1985; Raeder, 2006) on the dayside magnetopause or at high latitudes. In this paper, we believe that we observe a reconnection jet different from a regular VIR jet. Yet, the FTE-type structure itself may be the result of two VIR X-lines. For example, in Section 3, we calculate the FTE cross-section size to be $2,160 \mathrm{~km}$ $\left(17.6 d_{i}\right)$. Comparing the peak-to-peak interval to the $\mathrm{KH}$ period, we refer the size of the FTE to be $3 \%$ of the $\mathrm{KH}$ wavelength which is in excellent agreement with the simulation fit from Nakamura et al. (2013). We next discuss other properties of the FTE that may be consistent with the vortex-induced flux ropes as follows.

The FTE reported here has a core magnetic field in the $Z_{G S E}$ or north-south direction, similar to those reported by Eriksson et al. (2009), Nakamura et al. (2013), and Tang et al. (2018). To obtain the axis of the flux rope-like structure, we have additionally performed MVA on the data interval in Figures $4 \mathrm{k}$ - 4o. The axial direction, taken as the intermediate eigenvector, is found to be $[0.245,0.208,0.947]$. This direction makes an angle of $13^{\circ}$ from the Z-direction of the magnetopause model obtained in Section 2. In our case, we found the maximum growth rate of the $\mathrm{KH}$ instability when the angle $\theta_{0}$ is $-11^{\circ}$, which translates to an angle of $11^{\circ}$ between the magnetic field and the Z-direction. Thus, the axial angle is in a good agreement with the expected growth angle of the tearing mode satisfying $\mathbf{k} \cdot \mathbf{B}=0$. This flux-rope orientation is different from typical FTEs that are observed on the dayside magnetopause which is rather oriented in the $Y_{G S E}$ or east-west direction. The difference in the principal axis direction implies that our FTE is not generated by magnetic reconnection at the dayside. It is more likely that our FTE is locally generated by the VIR which has a strong guide-field in the $Z_{G S E}$ direction as suggested in the simulations by Nakamura et al. $(2011,2013)$. The presence of a strong IMF $B_{Y}$ may also lead to a magnetic flux rope with the core field in $Z$ direction (e.g. Øieroset et al., 2019) but this does not apply to our case because the IMF is strongly northward.

One may expect the strength of the core magnetic field to be much larger than the magnitude of the bipolar magnetic field variation of the FTE owing to the strong guide field expected for reconnection within $\mathrm{KH}$ vortices. In Eriksson et al. (2016), the guide field magnitude is 4 times the reconnecting component magnitude. We may argue that the core field strength should be dependent on the background (guide) field value. In our case, the background field is about 1.5 times the in-plane field variations (see Figure 3). The FTE core field strength $\left(B_{M}\right)$ increases up to 2.5 times the bipolar variation magnitude $\left(\left|B_{L}\right|\right)$ as seen in Figure 5. This core field is thus $67 \%$ enhanced from the background value. For comparison, Nakamura et al. (2011) observed that the core field during the bipolar fluctuation to be $30 \%$ above the background value. The enhanced core field of twice the expected value may be related to the magnetic topology of the FTE as we investigate next.

We now consider the magnetic topology of our FTE. Figure 6 shows the electron pitch angle distribution (ePAD) in panel (a) and electron parallel heat flux in panel (b) between 20:06:36 and 20:07:10 UT, covering the FTE interval between 20:06:46 and 20:06:56 shown in Figures 4 and 5. The ePAD is shown in the suprathermal energy range $420-726 \mathrm{eV}$, which is considered typical for magnetosheath acceleration (e.g. Lavraud et al., 2005; Lavraud et al., 2006; Pu et al., 2013; Zhong et al., 2013; Kacem et al., 2018) and provides a good tracer of magnetic field topology. The FTE reconnecting current sheet is at 20:06:51.2 UT $\left(t_{c}\right)$, marked by a vertical dashed line. The ePAD phase space density shows peaks at both $0^{\circ}$ and $180^{\circ}$ before $t_{c}$ while it mainly peaks 


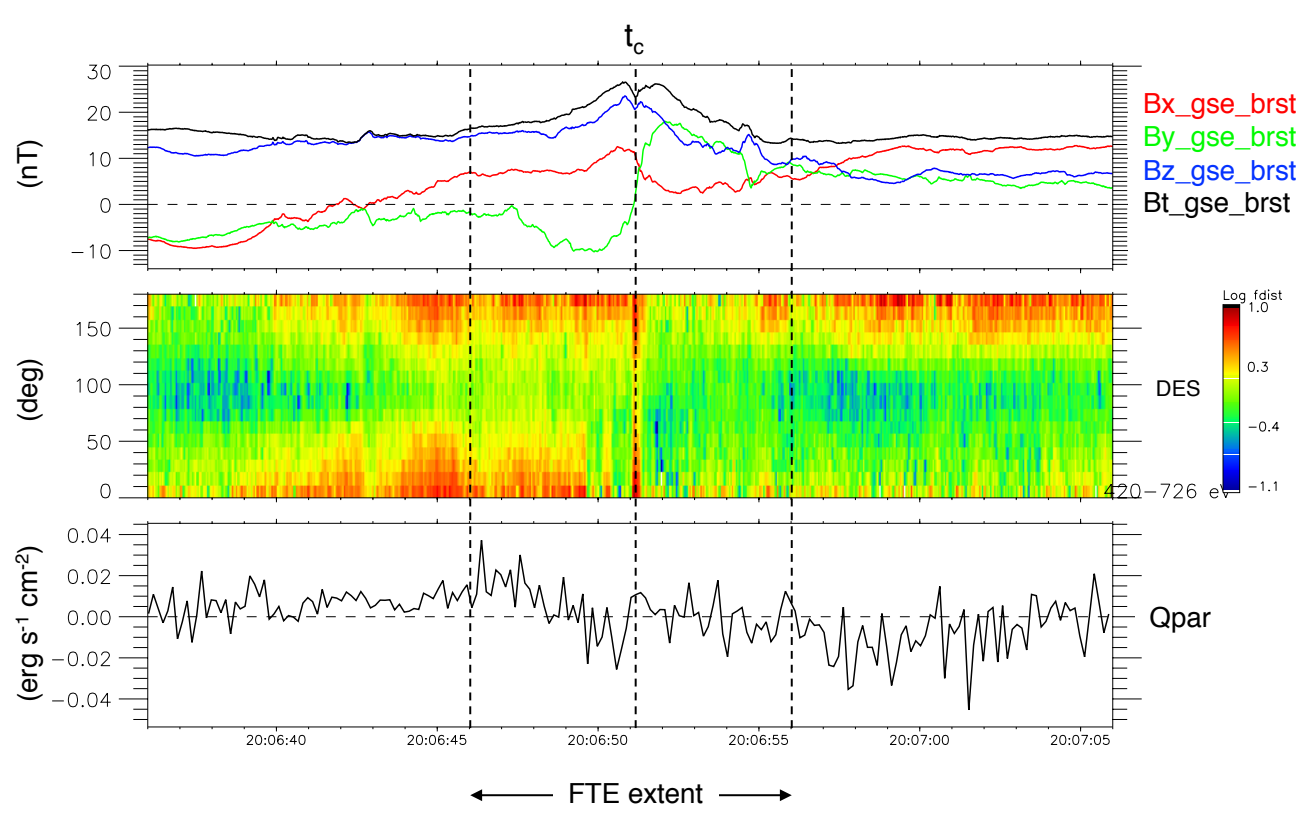

Figure 6. MMS 1 data between between 20:06:36 and 20:07:06 UT showing (a) magnetic fields, (b) electron pitch angle distribution in the range of $420-726 \mathrm{eV}$, and (c) electron parallel heat flux.

at $180^{\circ}$ after $t_{c}$. The electron parallel heat flux is mainly positive with an average value about $0.01 \mathrm{erg} \mathrm{s}^{-1} \mathrm{~cm}^{-2}$ before $t_{c}$ while it is mainly negative with an average value about $-0.02 \mathrm{erg} \mathrm{s}^{-1} \mathrm{~cm}^{-2}$ after $t_{c}$, consistent with the predominance of electron phase space density at $180^{\circ}$ pitch angle. These indicate that the electron populations on either side of the FTE current sheet is associated with different magnetic field topologies. The difference in electron temperature on either side of the current sheet seen in Figure 4f may also support this interpretation. An FTE with different ePAD on either side of the FTE centre with an ion jet in between was reported by Kacem et al. (2018). They interpreted that, unlike a classical FTE structure, their FTE is more consistent with two different interlacing flux tubes with an ongoing magnetic reconnection at their interface. Øieroset et al. (2019) also reported a similar case. Our FTE is consistent with this interpretation.

Using 3-D kinetic simulations, Daughton et al. (2011) show that the tearing instability can generate multiple oblique flux ropes in which their formation and interaction were shown to drive turbulence. Nakamura et al. (2013); Daughton et al. (2014) show that the tearing mode can also develop within the compressed current sheet at trailing edges of KH waves. The process produces magnetic flux ropes along the periphery of the KH vortices which then get carried along with the shear and vortical flow. In the simulations, a flux rope produced at the stagnation (i.e., hyperbolic) point on a trailing edge aligns almost perfectly along the Z-direction. However, further away from the stagnation point, i.e., at the vortex top (the wave trough), the flux rope becomes tilted with $10^{\circ}$ oblique angle away from the Z-axis. This angle increases to $21^{\circ}$ at farther locations. Based on this picture, we may project that the tilted flux ropes from different locations along the compressed current sheet can have a shear angle relative to each other. This process, if assumed to evolve further, would lead to entangled flux 
ropes (see their Figure 5a). Further interaction between different flux ropes, which was not discussed in Nakamura et al. (2013), may occur as well. Kacem et al. (2018) showed a reconnection event at the interface of interlaced flux tubes with a relative shear angle of $73^{\circ}$. Øieroset et al. (2019) also found a similar case with a shear angle of $37^{\circ}$. Considering the event of Kacem et al. (2018) and similar events, Øieroset et al. (2019) further discussed that the flux tubes should continue interlacing and enhancing the magnetic shear of the current sheet up to $90^{\circ}$ (see their Figure 11). This is because the two tangled flux tubes tend to rotate to be more perpendicular to each other. In our case, the magnetic shear angle across the current sheet in Figure 5 is found to be $49^{\circ} \pm 1^{\circ}$. Our results may imply further evolution of the flux ropes produced from the $\mathrm{VIR}$ in $\mathrm{KH}$ waves.

Based on our analyses above, we propose a scenario for the observations in Figure 5. First, we illustrate the configurations of (a) type-I VIR and (b) our proposed reconnection at the interface between two interlaced flux tubes likely produced from multiple VIR X-lines in Figure 7. Here we use estimated current sheet coordinates of the CS1 - CS4, and the FTE-CS in Figure 3, respectively, derived from the hybrid MVA method (see Sections 2 and 4). For typical type-I VIR, a compressed current sheet (red dashed line) would form along the KH trailing edge (black solid line), giving its normal (red arrow) perpendicular to the trailing edge and with the VIR jets (green arrows) directed along the trailing edge. For our proposed scenario, however, type-I VIR is induced at multiple locations along the $\mathrm{KH}$ trailing edge, e.g., labelled here as RX1 and RX2. Due to adjacent locations of the multiple X-lines, RX1 and RX2 can produce VIR jets (shown as $\Delta V_{N}>0$ and $\Delta V_{N}<0$ in the FTE-CS coordinates) toward each other, carrying along the newly reconnected field lines (blue and pink arrows). Consequently, a secondary current sheet (orange dashed line) can form in between the converging jets (green arrows), with its normal (orange arrow) aligned with the $\mathrm{KH}$ trailing edge. Thus, the secondary jet (purple arrow, $\Delta V_{L}>0$ ) is detected perpendicular to the $\mathrm{KH}$ trailing edge at the interface between two flux tubes. Note that the main magnetic field $\left(B_{M}\right)$ is out-of-plane. The interlaced flux tubes are depicted with a $3-\mathrm{D}$ perspective as shown in the grey box (courtesy of Øieroset et al. (2019)).

Reconnection at the interface between flux tubes, presumably produced by VIR, at the KH wave trailing edge was previously unknown. This may be a new mechanism that contributes to the solar wind plasma mixing as mediated by $\mathrm{KH}$ waves at the magnetopause. Reconnection at the current sheet between two flux tubes making a shear angle is allowed by the condition $\Delta \beta<2\left(L / d_{i}\right) \tan (\theta / 2)$ (Swisdak et al., 2010), where $\Delta \beta$ is the difference in plasma- $\beta$ between the two inflow regions, $L / d_{i}$ is the width of the current sheet in units of ion inertial length, and $\theta$ is the magnetic shear angle. Øieroset et al. (2019) showed that, for a current sheet separating two flux tubes, magnetic flux pileup (which increases the shear angle $\theta$ and decreases $\Delta \beta$ ) at the interface of interlaced flux tubes is a necessary condition that allows reconnection with a low shear angle (i.e., less than $90^{\circ}$ ). The magnetic field pileup/enhancement surrounding the current sheet can also be seen in our case in Figure 6a, hence supporting the magnetic pileup condition of reconnection with a low shear angle. This suggests that magnetic flux tubes generated by VIR can reconnect when Swisdak et al. (2010)'s condition is satisfied. However, further studies are needed to estimate the contribution of this mechanism. More observations of such cases in magnetopause KH waves are desirable.

2-D simulations showed that two magnetic islands can coalesce to become a larger island (e.g. Oka et al., 2010). For two coalescing flux ropes, one may expect a pair of bipolar magnetic field variations instead of a single bipolar variation. Øieroset et al. (2016) proposed a scenario that could explain the single bipolar magnetic field variation, namely that the current sheet at the centre of an FTE was formed between 
(a)

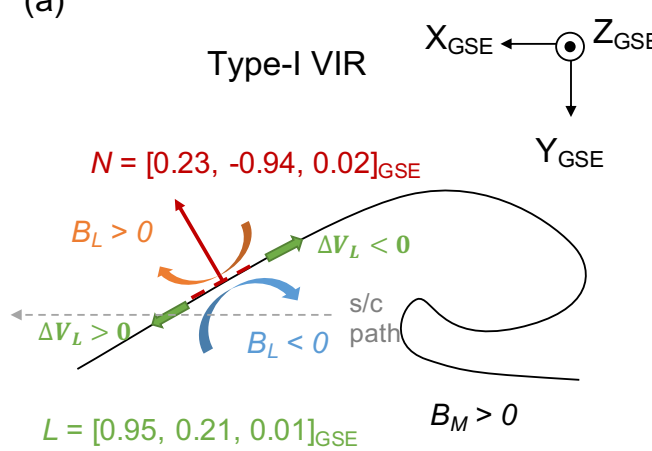

(b)

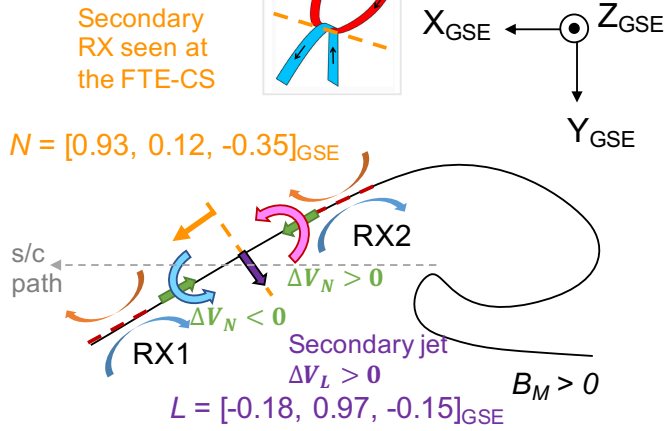

Figure 7. Schematic illustrations of (a) type-I vortex-induced reconnection (VIR) and (b) reconnection at the interface between two interlaced flux tubes likely produced from multiple type-I VIR X-lines, with interlinked flux tubes in a 3-D perspective (grey box, adapted from (Øieroset et al., 2019)). In panel (a), we show the averaged $N, L$ directions of the current sheets marked in Figure 3 (red dashed line). In panel (b), we show the $N, L$ directions of the FTE current sheet (orange dashed line) in Figure 5 that are perpendicular to those in (a). A plausible explanation of this distinct orientation of the FTE current sheet is that it is formed due to two adjacent type-I VIR X-lines which produce jets (green arrows) converging toward each other, carrying along two newly reconnected field lines (blue and pink arrows). Consequently, a secondary reconnection jet (purple arrow) is produced due to the magnetic pileup in between the two interlaced flux tubes, with the direction perpendicular to the $\mathrm{KH}$ trailing edge. See also text for full descriptions.

field lines carried by converging jets from two X-lines. This scenario is plausible in 3-D because the field lines do not form closed loops. Zhou et al. (2017) reported evidence of two flux ropes undergoing coalescence but a pair of bipolar variations was again not observed. Instead, they observed a large bipolar and nearly symmetric variation with an embedded small, reversed bipolar variation at the centre of the large variation (called a 'quadrupolar' signature in their paper). By comparing with a particle-in-cell simulation, they found that the small, reversed bipolar signature at the current sheet centre was a result of the dissipation or erosion of magnetic field at the interface of two merging flux ropes. In contrast, a more symmetric pair of bipolar variations would be observed for two adjacent flux ropes with no interaction and dissipation (Zhou et al., 2017). Only one bipolar variation was also observed in Kacem et al. (2018) and Øieroset et al. (2019). The absence of the small, reversed bipolar signature at the centre in our case and similar cases may imply that the two flux ropes have significantly evolved and merged after the dissipation phase.

Finally, we note that there is only one obvious fully-developed FTE signature for the whole $\mathrm{KH}$-active interval of a few hours studied here. There may be another FTE in this KH event albeit for a possibly small one (in magnetic field magnitude) at 20:02 UT with no sign of reconnection at its core. There is none in the event studied by Eriksson et al. (2016) and Vernisse et al. (2016). Thus, it is likely that this kind of event is rare. More observations would be desirable to conclude on the rarity of VIR-induced flux ropes and interlaced flux tubes as reported in our case. 


\section{Summary and Conclusions}

We report MMS observations of an FTE during the $\mathrm{KH}$ event detected on 5 May 2017 at the dawnside flank magnetopause beyond the post-terminator. The FTE structure is characterised by the bipolar $B_{Y}$ variation, enhanced magnetic core field $B_{Z}$, and enhanced total pressure dominated by magnetic pressure that are embedded in the large-scale variations subject to the KH waves. The curvature variation clearly resembles the passage of a cylinder-like magnetic structure in a cross-section wise direction. The FTE signatures appear during the spacecraft crossing of a trailing (sunward facing) edge of the KH waves. The cross-section scale size of the FTE is found to be $2,160 \mathrm{~km}$ using the minimum curvature radius near the FTE centre. The ratio of the peak-to-peak interval of the bipolar $B_{Y}$ variation to the $\mathrm{KH}$ period is observed to be $0.03 \pm 0.01$. The orientation, core field enhancement, and size of this FTE are consistent with flux ropes that are produced from multiple VIR along the periphery of KH waves as predicted in the simulations by Nakamura et al. (2011, 2013).

At the centre of the bipolar $B_{Y}$ variation (the FTE current sheet), an ion jet with $\Delta V_{Y}>0$ is observed. The ion jet has a positive correlation with the $B_{Y}$ variation on one side and a negative correlation on the other side, consistent with Walén relation which implies that this ion jet might be produced from reconnection (e.g., Phan et al., 2004). However, the observed ion jet speed is $50 \%$ lower than the predicted speed. We attributed the lower ion jet speed to be due to the proximity to the X-line (Phan et al., 2016). Other properties at the current sheet centre that support the interpretation of reconnection include:

- the enhancement in particle number densities $\left(\sim 3 \mathrm{~cm}^{-3}\right)$ that are expected for an exhaust region being populated by plasmas from the inflow regions,

- the electron parallel heating $(\sim 50 \mathrm{eV})$ that is expected for the reconnection exhaust in a strong guide-field environment,

- the dip in magnetic field strength $(\sim 3 \mathrm{nT})$ that is consistent with the magnetic field energy conversion due to reconnection, and

- the small variation in the guide field direction $\left(\left|\Delta B_{M}\right| \sim 2.6 \mathrm{nT}\right)$, in addition to the FTE enhanced core field, that resembles the Hall magnetic field pattern.

Based on the ePAD and electron parallel heat flux, we further found that the magnetic fields on either side of the FTE current sheet are topologically unconnected. Hence, our FTE is inconsistent with a single flux rope, but rather consistent with two interlacing flux tubes and with reconnection at the interface as reported by Kacem et al. (2018). In Nakamura et al. (2013), VIR-produced FTEs can become tilted away from the northward direction (due to the oblique tearing mode) with different angles depending on the location along the periphery of the KH waves. We thus project that the interlaced flux tubes found in our case may be a later stage of the evolution of these FTEs. In our case, we found that the two flux tubes make an angle of about $50^{\circ}$ to each other. Reconnection at the current sheet separating two flux tubes with a low shear angle is facilitated by the magnetic field pileup near the current sheet centre (Øieroset et al., 2019), which is also observed here. Based on observations and analyses, we proposed a scenario leading to reconnection at the interface between interlaced flux tubes likely produced from multiple type-I VIR X-lines as shown in Figure 7b. This mechanism may also contribute to the solar wind plasma mixing as mediated by magnetopause KH waves. There is still some uncertainty as to whether the observed reconnection is due to colliding jets at the center of a previously formed FTE, or the result of the interaction of distinct flux tubes as proposed here (though the latter is preferred given the different suprathermal electron properties). Finally, we note that there is only one obvious fully-developed FTE for the entire KH event, thus this kind of event should be rare. More observations would be desirable for a better 
understanding of the interaction between vortex-induced flux ropes and the rarity of such event.

\section{Acknowledgments}

R. K. acknowledges financial support from Junior Research Fellowship Program 2019, Embassy of France in Thailand, and University of Toulouse (III), Paul Sabatier, France. Thesis work by R.K. was funded by CEMPS at the University of Exeter. Work at IRAP is supported by CNRS and CNES. C.F. acknowledges financial support from the UK Science and Technology Facilities Council (STFC) under her Advanced Fellowship ST/I003649. K. M. and D. R. acknowledge financial support from Thailand Science Research and Innovation via grants MRG6180176 and RTA6280002. M.Ø. acknowledges NASA grants NNX17AE12G and 80NSSC18K1380. MMS data are available from https://lasp.colorado.edu/mms/sdc/public/. We visualise data using CLWeb software available at http://clweb.irap.omp.eu/cl/clweb.php.

\section{References}

Borgogno, D., Califano, F., Faganello, M., \& Pegoraro, F. (2015, March). Doublereconnected magnetic structures driven by Kelvin-Helmholtz vortices at the Earth's magnetosphere. Phys. Plasmas, 22, 032301. doi: 10.1063/1.4913578

Bothmer, V., \& Schwenn, R. (1998, January). The structure and origin of magnetic clouds in the solar wind. Ann. Geophys., 16, 1-24. doi: 10.1007/s00585-997 $-0001-\mathrm{x}$

Burch, J., Moore, T. E., Torbert, R., \& Giles, B. L. (2015). Magnetospheric Multiscale Overview and Science Objectives. Sp. Sci. Rev., 199, 5-21. doi: 10.1007/ s11214-015-0164-9

Chandrasekhar, S. (1961). Hydrodynamic and hydromagnetic stability. Clarendon Press.

Chaston, C. C., Wilber, M., Mozer, F. S., Fujimoto, M., Goldstein, M. L., Acuna, M., ... Fazakerley, A. (2007). Mode conversion and anomalous transport in Kelvin-Helmholtz vortices and kinetic Alfvén waves at the earth's magnetopause. Phys. Rev. Lett., 99(17), 1-4. doi: 10.1103/PhysRevLett.99.175004

Daughton, W., Nakamura, T. K. M., Karimabadi, H., Roytershteyn, V., \& Loring, B. (2014, May). Computing the reconnection rate in turbulent kinetic layers by using electron mixing to identify topology. Physics of Plasmas, 21 (5), 052307. doi: 10.1063/1.4875730

Daughton, W., Roytershteyn, V., Karimabadi, H., Yin, L., Albright, B. J., Bergen, B., \& Bowers, K. J. (2011, Jul). Role of electron physics in the development of turbulent magnetic reconnection in collisionless plasmas. Nature Physics, 7(7), 539-542. doi: $10.1038 /$ nphys 1965

Dunlop, M. W., Southwood, D. J., Glassmeier, K. H., \& Neubauer, F. M. (1988). Analysis of multipoint magnetometer data. Adv. Sp. Res., 8(9-10), 273-277. doi: 10.1016/0273-1177(88)90141-X

Eastwood, J. P., Shay, M. A., Phan, T. D., \& Øieroset, M. (2010, May). Asymmetry of the Ion Diffusion Region Hall Electric and Magnetic Fields during Guide Field Reconnection: Observations and Comparison with Simulations. Phys. Rev. Lett., 104(20), 205001. doi: 10.1103/PhysRevLett.104.205001

Eriksson, S., Hasegawa, H., Teh, W.-L., Sonnerup, B. U. Ö., McFadden, J. P., Glassmeier, K.-H., ... Carlson, C. W. (2009). Magnetic island formation between large-scale flow vortices at an undulating postnoon magnetopause for northward interplanetary magnetic field. J. Geophys. Res., 114(A1). doi: 10.1029/2008JA013505

Eriksson, S., Lavraud, B., Wilder, F. D., Stawarz, J. E., Giles, B. L., Burch, J. L., ... Goodrich, K. A. (2016). Magnetospheric Multiscale observations of mag- 
netic reconnection associated with Kelvin-Helmholtz waves.

Geophys. Res.

Lett., 43(11), 5606-5615. doi: 10.1002/2016GL068783

Eriksson, S., Wilder, F. D., Ergun, R. E., Schwartz, S. J., Cassak, P. A., Burch, J. L., ... Marklund, G. T. (2016, Jul). Magnetospheric Multiscale Observations of the Electron Diffusion Region of Large Guide Field Magnetic Reconnection. Phys. Rev. Lett., 117(1), 015001. doi: 10.1103/ PhysRevLett.117.015001

Faganello, M., Califano, F., Pegoraro, F., \& Andreussi, T. (2012). Double midlatitude dynamical reconnection at the magnetopause: An efficient mechanism allowing solar wind to enter the Earth's magnetosphere. Europhys. Lett., 100 (6). doi: 10.1209/0295-5075/100/69001

Farrugia, C. J., Gratton, F. T., Bender, L., Biernat, H. K., Erkaev, N. V., Quinn, J. M., .. Dennisenko, V. (1998). Charts of joint Kelvin-Helmholtz and Rayleigh-Taylor instabilites at the dayside magnetopause for strongly northward interplanetary magnetic field. J. Geophys. Res., 103(A4), 6703. doi: 10.1029/97JA03248

Farrugia, C. J., Gratton, F. T., Contin, J., Cocheci, C. C., Arnoldy, R. L., Ogilvie, K. W., ... Rostoker, G. (2000, Apr). Coordinated Wind, Interball/tail, and ground observations of Kelvin-Helmholtz waves at the near-tail, equatorial magnetopause at dusk: January 11, $1997 . \quad$ J. Geophys. Res., 105(A4), 76397668. doi: 10.1029/1999JA000267

Farrugia, C. J., Rijnbeek, R. P., Saunders, M. A., Southwood, D. J., Rodgers, D. J., Smith, M. F., .. Woolliscroft, L. J. C. (1988). A multi-instrument study of flux transfer event structure. J. Geophys. Res., 93(A12), 14465-14477. doi: 10.1029/JA093iA12p14465

Gosling, J. T., \& Phan, T. D. (2013, Feb). Magnetic Reconnection in the Solar Wind at Current Sheets Associated with Extremely Small Field Shear Angles. Astrophys. J. Lett., 763(2), L39. doi: 10.1088/2041-8205/763/2/L39

Gosling, J. T., Skoug, R. M., McComas, D. J., \& Smith, C. W. (2005, Jan). Direct evidence for magnetic reconnection in the solar wind near 1 AU. J. Geophys. Res., 110(A1), A01107. doi: 10.1029/2004JA010809

Haggerty, C. C., Shay, M. A., Chasapis, A., Phan, T. D., Drake, J. F., Malakit, K., ... Kieokaew, R. (2018, Oct). The reduction of magnetic reconnection outflow jets to sub-Alfvénic speeds. Physics of Plasmas, 25(10), 102120. doi: $10.1063 / 1.5050530$

Hudson, P. D. (1970, Nov). Discontinuities in an anisotropic plasma and their identification in the solar wind. Planet. Space Sci., 18(11), 1611-1622. doi: 10 .1016/0032-0633(70)90036-X

Kacem, I., Jacquey, C., Génot, V., Lavraud, B., Vernisse, Y., Marchaudon, A., ... Penou, E. (2018). Magnetic reconnection at a thin current sheet separating two interlaced flux tubes at the earth's magnetopause. J. Geophys. Res., 123(3), 1779-1793. doi: 10.1002/2017JA024537

Kawano, H., \& Russell, C. T. (1996, Dec). Survey of flux transfer events observed with the ISEE 1 spacecraft: Rotational polarity and the source region. J. Geophys. Res., 101 (A12), 27299-27308. doi: 10.1029/96JA02703

Kieokaew, R. (2019). Multi-scale Structure Analyses of Magnetopause KelvinHelmholtz Waves: Applications of Four-spacecraft to MHD Simulations and Cluster and MMS Observations (Unpublished doctoral dissertation). University of Exeter.

Kieokaew, R., \& Foullon, C. (2019, May). Kelvin-Helmholtz waves magnetic curvature and vorticity: four-spacecraft Cluster observations. J. Geophys. Res., 124, 3347- 3359. doi: 10.1029/2019JA026484

Kieokaew, R., Foullon, C., \& Lavraud, B. (2018, Jan). Four-spacecraft magnetic curvature and vorticity analyses on Kelvin-Helmholtz waves in MHD simulations. J. Geophys. Res., 123, 513-529. doi: 10.1002/2017JA024424 
King, J. H., \& Papitashvili, N. E. (2005, Feb). Solar wind spatial scales in and comparisons of hourly Wind and ACE plasma and magnetic field data. J. Geophys. Res., 110(A2), A02104. doi: 10.1029/2004JA010649

Lavraud, B., Thomsen, M. F., Lefebvre, B., Schwartz, S. J., Seki, K., Phan, T. D., ... Rème, H. (2006). Evidence for newly closed magnetosheath field lines at the dayside magnetopause under northward IMF. J. Geophys. Res., 111, A05211. doi: 10.1029/2005JA011266

Lavraud, B., Thomsen, M. F., Taylor, M. G. G. T., Wang, Y. L., Phan, T. D., Schwartz, S. J., .. Rème, H. (2005). Characteristics of the magnetosheath electron boundary layer under northward interplanetary magnetic field : Implications for high-latitude reconnection. J. Geophys. Res., 110, 1-9. doi: 10.1029/2004JA010808

Lavraud, B., Zhang, Y. C., Vernisse, Y., Gershman, D. J., Dorelli, J., Cassak, P. A., ... Yokota, S. (2016, Apr). Currents and associated electron scattering and bouncing near the diffusion region at Earth's magnetopause. Geophys. Res. Lett., 43(7), 3042-3050. doi: 10.1002/2016GL068359

Lee, L. C., \& Fu, Z. F. (1985, February). A theory of magnetic flux transfer at the Earth's magnetopause. Geophys. Res. Lett., 12, 105-108. doi: 10.1029/ GL012i002p00105

Li, W. Y., André, M., Khotyaintsev, Y. V., Vaivads, A., Graham, D. B., ToledoRedondo, S., ... Strangeway, R. J. (2016). Kinetic evidence of magnetic reconnection due to Kelvin-Helmholtz waves. Geophys. Res. Lett., 43(11), 5635-5643. doi: 10.1002/2016GL069192

Matsumoto, Y., \& Hoshino, M. (2006). Turbulent mixing and transport of collisionless plasmas across a stratified velocity shear layer. J. Geophys. Res., 111(A5). Retrieved from https://agupubs.onlinelibrary.wiley.com/doi/ abs/10.1029/2004JA010988 doi: 10.1029/2004JA010988

Nakamura, T. K. M., Daughton, W., Karimabadi, H., \& Eriksson, S. （2013, Sep). Three-dimensional dynamics of vortex-induced reconnection and comparison with THEMIS observations. J. Geophys. Res., 118, 5742-5757. doi: 10.1002/jgra.50547

Nakamura, T. K. M., Fujimoto, M., \& Otto, A. (2006, July). Magnetic reconnection induced by weak Kelvin-Helmholtz instability and the formation of the low-latitude boundary layer. Geophys. Res. Lett., 33, L14106. doi: 10.1029/2006GL026318

Nakamura, T. K. M., Hasegawa, I., H. Shinohara, \& Fujimoto, M. (2011). Evolution of an MHD-scale Kelvin-Helmholtz vortex accompanied by magnetic reconnection: Two-dimensional particle simulations. J. Geophys. Res., 116(3), 1-18. doi: 10.1029/2010JA016046

Nykyri, K., \& Otto, A. (2001). Plasma transport at the magnetospheric boundary due to reconnection in Kelvin-Helmholtz vortices. Geophys. Res. Lett., 28(18), $3565-3568$.

Nykyri, K., Otto, A., Lavraud, B., Mouikis, C., Kistler, L. M., Balogh, A., \& Rème, H. (2006, October). Cluster observations of reconnection due to the KelvinHelmholtz instability at the dawnside magnetospheric flank. Ann. Geophys., 24, 2619-2643.

Ogilvie, K. W., \& Fitzenreiter, R. J. (1989, November). The Kelvin-Helmholtz instability at the magnetopause and inner boundary layer surface. J. Geophys. Res., 94, 15113-15123. doi: 10.1029/JA094iA11p15113

Øieroset, M., Phan, T. D., Drake, J. F., Eastwood, J. P., Fuselier, S. A., Strangeway, R. J., ... Paterson, W. (2019). Reconnection with magnetic flux pileup at the interface of converging jets at the magnetopause. Geophys. Res. Lett., 46(4), 1937-1946. doi: 10.1029/2018GL080994

Øieroset, M., Phan, T. D., Haggerty, C., Shay, M. A., Eastwood, J. P., Gershman, D. J., ... Malakit, K. (2016). Mms observations of large guide field symmetric 
reconnection between colliding reconnection jets at the center of a magnetic flux rope at the magnetopause. Geophys. Res. Lett., 43(11), 5536-5544. doi: 10.1002/2016GL069166

Øieroset, M., Phan, T. D., Lin, R. P., \& Sonnerup, B. U. Ö. (2000, Nov). Walén and variance analyses of high-speed flows observed by Wind in the midtail plasma sheet: Evidence for reconnection. J. Geophys. Res., 105(A11), 25247-25264. doi: 10.1029/2000JA900075

Oka, M., Phan, T. D., Krucker, S., Fujimoto, M., \& Shinohara, I. (2010, May). Electron Acceleration by Multi-Island Coalescence. Astrophys. J., 714 (1), 915-926. doi: 10.1088/0004-637X/714/1/915

Otto, A., \& Fairfield, D. H. (2000, September). Kelvin-Helmholtz instability at the magnetotail boundary: MHD simulation and comparison with Geotail observations. J. Geophys. Res., 105, 21. doi: 10.1029/1999JA000312

Paschmann, G., Haerendel, G., Papamastorakis, I., Sckopke, N., Bame, S. J., Gosling, J. T., \& Russell, C. T. (1982). Plasma and magnetic field characteristics of magnetic flux transfer events. J. Geophys. Res., 87(A4), 2159. doi: 10.1029/JA087iA04p02159

Paschmann, G., Papamastorakis, I., Baumjohann, W., Sckopke, N., Carlson, C. W., Sonnerup, B. U. Ö., \& Lühr, H. (1986, Oct). The magnetopause for large magnetic shear: AMPTE/IRM observations. J. Geophys. Res., 91 (A10), 11099-11115. doi: 10.1029/JA091iA10p11099

Phan, T. D., Drake, J. F., Shay, M. A., Mozer, F. S., \& Eastwood, J. P. (2007, Dec). Evidence for an Elongated (\&gt;60 Ion Skin Depths) Electron Diffusion Region during Fast Magnetic Reconnection. Phys. Rev. Lett., 99(25), 255002. doi: 10.1103/PhysRevLett.99.255002

Phan, T. D., Dunlop, M. W., Paschmann, G., Klecker, B., Bosqued, J. M., Rème, H., ... Kistler, L. M. (2004). Cluster observations of continuous reconnection at the magnetopause under steady interplanetary magnetic field conditions. Ann. Geophys., 22(7), 2355-2367. doi: 10.5194/angeo-22-2355-2004

Phan, T. D., Eastwood, J. P., Cassak, P. A., Øieroset, M., Gosling, J. T., Gershman, D. J., ... Wilder, F. D. (2016). MMS observations of electron-scale filamentary currents in the reconnection exhaust and near the X line. Geophys. Res. Lett., 43(12), 6060-6069. doi: 10.1002/2016GL069212

Phan, T. D., Paschmann, G., \& Sonnerup, B. U. Ö. (1996, Apr). Low-latitude dayside magnetopause and boundary layer for high magnetic shear 2. Occurrence of magnetic reconnection. J. Geophys. Res., $101(\mathrm{~A} 4), 7817-7828$. doi: 10.1029/95JA03751

Phan, T. D., Shay, M. A., Haggerty, C. C., Gosling, J. T., Eastwood, J. P., Fujimoto, M., ... Angelopoulos, V. (2016). Ion Larmor radius effects near a reconnection $\mathrm{X}$ line at the magnetopause: THEMIS observations and simulation comparison. Geophys. Res. Lett., 43(17), 8844-8852. doi: 10.1002/2016GL070224

Pollock, C., Moore, T., Jacques, A., Burch, J., Gliese, U., Saito, Y., ... Zeuch, M. (2016). Fast Plasma Investigation for Magnetospheric Multiscale. Space Sci. Rev., 199, 331-406. doi: 10.1007/s11214-016-0245-4

Pu, Z. Y., Raeder, J., Zhong, J., Bogdanova, Y. V., Dunlop, M., Xiao, C. J., ... Fazakerley, A. (2013). Magnetic topologies of an in vivo FTE observed by Double Star/TC-1 at Earth's magnetopause. Geophys. Res. Lett., $40(14)$, 3502-3506. doi: 10.1002/grl.50714

Raeder, J. (2006, March). Flux Transfer Events: 1. generation mechanism for strong southward IMF. Ann. Geophys., 24, 381-392. doi: 10.5194/angeo-24-381-2006

Rijnbeek, R. P., Cowley, S. W. H., Southwood, D. J., \& Russell, C. T. (1984, February). A survey of dayside transfer events observed by ISEE 1 and 2 magnetometers. J. Geophys. Res., 89, 786-800. doi: 10.1029/JA089iA02p00786

Robert, P., Roux, A., Harvey, C. C., Dunlop, M. W., Daly, P. W., \& Glassmeier, 
K.-H. (1998). Tetrahedron Geometric Factors. ISSI Sci. Reports Ser., 001, $323-328$.

Rossi, C. (2015). Kelvin-Helmholtz Instability at the Magnetopause: Theory and Observations (Unpublished doctoral dissertation). Université Pierre et Marie Curie - Paris VI and Universitá Degli Studi Di Pisa.

Runov, A., Nakamura, R., Baumjohann, W., Treumann, R. A., Zhang, T. L., Volwerk, M., ... Kistler, L. (2003, Jun). Current sheet structure near magnetic X-line observed by Cluster. Geophys. Res. Lett., 30(11), 10-13. doi: 10.1029/2002GL016730

Runov, A., Sergeev, V. A., Baumjohann, W., Nakamura, R., Apatenkov, S., Asano, Y., ... Rème, H. (2005, June). Electric current and magnetic field geometry in flapping magnetotail current sheets. Ann. Geophys., 23, 1391-1403. doi: 10.5194/angeo-23-1391-2005

Russell, C. T., Anderson, B. J., Baumjohann, W., Bromund, K. R., Dearborn, D., Fischer, D., ... Richter, I. (2016, March). The Magnetospheric Multiscale Magnetometers. Space Sci. Rev., 199, 189-256. doi: 10.1007/ s11214-014-0057-3

Russell, C. T., \& Elphic, R. C. (1978). Initial ISEE Magnetometer Results: Magnetopause Observations. Space Sci. Rev., 22, 681-715.

Russell, C. T., \& Elphic, R. C. (1979, January). ISEE observations of flux transfer events at the dayside magnetopause. Geophys. Res. Lett., 6, 33-36. doi: 10 .1029/GL006i001p00033

Russell, C. T., Le, G., \& Kuo, H. (1996). The occurrence rate of flux transfer events. Adv. Sp. Res., 18(8), 197-205. doi: 10.1016/0273-1177(95)00965-5

Russell, C. T., Mellott, M. M., Smith, E. J., \& King, J. H. (1983). Multiple spacecraft observations of interplanetary shocks: Four spacecraft determination of shock normals. J. Geophys. Res., 88(A6), 4739-4748. doi: 10.1029/JA088iA06p04739

Sanny, J., Sibeck, D. G., Venturini, C. C., \& Russell, C. T. (1996, Mar). A statistical study of transient events in the outer dayside magnetosphere. J. Geophys. Res., 101(A3), 4939-4952. doi: 10.1029/95JA03063

Saunders, M. A., Russell, C. T., \& Sckopke, N. $\quad$ (1984, February). Flux transfer events: Scale size and interior structure. Geophys. Res. Lett., 11, 131-134. doi: 10.1029/GL011i002p00131

Scholer, M. (1988, April). Magnetic flux transfer at the magnetopause based on single X line bursty reconnection. Geophys. Res. Lett., 15, 291-294. doi: 10.1029/ GL015i004p00291

Sckopke, N., Paschmann, G., Haerendel, G., Sonnerup, B. U. O., Bame, S. J., Forbes, T. G., .. Russell, C. T. (1981, April). Structure of the lowlatitude boundary layer. J. Geophys. Res., 86, 2099-2110. doi: 10.1029/ JA086iA04p02099

Shen, C., Li, X., Dunlop, M., Liu, Z. X., Balogh, A., Baker, D. N., ... Wang, X. (2003). Analyses on the geometrical structure of magnetic field in the current sheet based on cluster measurements. J. Geophys. Res., 108(A5). doi: 10.1029/2002JA009612

Shue, J.-H., Chao, J. K., Fu, H. C., Russell, C. T., Song, P., Khurana, K. K., \& Singer, H. J. (1997). A new functional form to study the solar wind control of the magnetopause size and shape. J. Geophys. Res., 102(A5), 9497-9511. doi: 10.1029/97JA00196

Sibeck, D. G., Kuznetsova, M., Angelopoulos, V., Glaßmeier, K.-H., \& McFadden, J. P. (2008). Crater ftes: Simulation results and themis observations. Geophys. Res. Lett., 35(17). doi: 10.1029/2008GL033568

Sonnerup, B. U. Ö. (1979). Magnetic field reconnection. In Solar system plasma physics (Vol. 3, p. 45-108). Amsterdam: North-Holland Publishing Co.

Sonnerup, B. U. Ö., \& Cahill, L. J. (1967). Magnetopause structure and attitude 
from Explorer 12 observations. J. Geophys. Res., 72(1), 171. doi: 10.1029/ JZ072i001p00171

Southwood, D. J., Farrugia, C. J., \& Saunders, M. A. (1988, May). What are flux transfer events? Planet. Space Sci., 36(5), 503-508. doi: 10.1016/0032-0633(88) 90109-2

Stawarz, J. E., Eriksson, S., Wilder, F. D., Ergun, R. E., Schwartz, S. J., Pouquet, A., ... Sturner, A. P. (2016, Nov). Observations of turbulence in a KelvinHelmholtz event on 8 September 2015 by the Magnetospheric Multiscale mission. J. Geophys. Res., 121(11), 11,021-11,034. doi: 10.1002/2016JA023458

Sturner, A. P., Eriksson, S., Nakamura, T., Gershman, D. J., Plaschke, F., Ergun, R. E., .. Burch, J. L. (2018). On Multiple Hall-Like Electron Currents and Tripolar Guide Magnetic Field Perturbations During Kelvin-Helmholtz Waves. J. Geophys. Res., 123(2), 1305-1324. doi: 10.1002/2017JA024155

Swisdak, M., Opher, M., Drake, J. F., \& Alouani Bibi, F. （2010, Feb). The Vector Direction of the Interstellar Magnetic Field Outside the Heliosphere. Astrophys. J., $710(2)$, 1769-1775. doi: 10.1088/0004-637X/710/2/1769

Tang, B., Li, W., Wang, C., Dai, L., Khotyaintsev, Y., Lindqvist, P.-A., .. . Burch, J. (2018, Jun). Magnetic depression and electron transport in an ion-scale flux rope associated with Kelvin-Helmholtz waves. Ann. Geophys., 36(3), 879-889. doi: 10.5194/angeo-36-879-2018

Vernisse, Y., Lavraud, B., Eriksson, S., Gershman, D. J., Dorelli, J., Pollock, C., ... Yokota, S. (2016, Oct). Signatures of complex magnetic topologies from multiple reconnection sites induced by Kelvin-Helmholtz instability. J. Geophys. Res., 121(10), 9926-9939. doi: 10.1002/2016JA023051

Wang, Y. L., Elphic, R. C., Lavraud, B., Taylor, M. G. G. T., Birn, J., Raeder, J., ... Friedel, R. H. (2005). Initial results of high-latitude magnetopause and low-latitude flank flux transfer events from 3 years of cluster observations. $J$. Geophys. Res., 110(A11). doi: 10.1029/2005JA011150

Yang, Y. Y., Shen, C., Zhang, Y. C., Rong, Z. J., Li, X., Dunlop, M., .. Rème, H. (2014). The force-free configuration of flux ropes in geomagnetotail: Cluster observations. J. Geophys. Res., 119(8), 6327-6341. doi: 10.1002/2013JA019642

Zhang, H., Kivelson, M. G., Khurana, K. K., McFadden, J., Walker, R. J., Angelopoulos, V., ... Auster, H. U. (2010). Evidence that crater flux transfer events are initial stages of typical flux transfer events. J. Geophys. Res., 115(A8). doi: 10.1029/2009JA015013

Zhong, J., Pu, Z. Y., Dunlop, M. W., Bogdanova, Y. V., Wang, X. G., Xiao, C. J., ... Eastwood, J. P. (2013). Three-dimensional magnetic flux rope structure formed by multiple sequential X-line reconnection at the magnetopause. $J$. Geophys. Res., 118(5), 1904-1911. doi: 10.1002/jgra.50281

Zhou, M., Berchem, J., Walker, R. J., El-Alaoui, M., Deng, X., Cazzola, E., ... Burch, J. L. (2017). Coalescence of Macroscopic Flux Ropes at the Subsolar Magnetopause: Magnetospheric Multiscale Observations. Phys. Rev. Lett., 119(5), 1-6. doi: 10.1103/PhysRevLett.119.055101 